\title{
¿DE VAlles o DE PunA? Discutiendo interacción A PARTIR DE LA CARACTERIZACIÓN COMPOSICIONAL DE CONJUNTOS CERÁMICOS. EL CASO DE Antofagasta de la Sierra, Puna Sur Argentina
}

\author{
Sara M. L. López Campeny ${ }^{1}$
}

\section{* Introducción: : Antofagasta de la sierra COMO ESPACIO DE INTERACCIÓN}

\begin{abstract}
En el marco de la problemática de interacciones entre poblaciones de la Puna de Antofagasta de la Sierra y los valles próximos, presentamos nueva evidencia cerámica, obtenida a partir de la caracterización composicional petrográfica y química - por corte delgado y análisis de activación neutrónica instrumental (AANI) - de un conjunto cerámico vinculado a estilos "valliserranos", recuperado en sitios puneños. Los perfiles composicionales obtenidos fueron comparados con piezas procedentes de sitios arqueológicos valliserranos (Hualfín), del Bolsón de Fiambalá y la región puneña de Chaschuil (Tinogasta), para las que se disponía de datos composicionales obtenidos previamente por otros investigadores. Los resultados alcanzados contribuyen a la discusión sobre áreas de producción y circulación de los conjuntos cerámicos en el pasado.
\end{abstract}

Palabras claves: interacción puna y valles - cerámica - caracterización petrográfica - análisis de activación neutrónica instrumental.

Abstract

Here, we presents new ceramic evidence obtained from the compositional petrographic and chemical characterization - by means of a thin cut and an instrumental neutron activation analysis (INAA)of a ceramic set linked to "valliserranos" styles, recovered in local sites.

The compositional profiles obtained are compared with pieces from archaeological sites of valley (Hualfín), in the Bolsón de Fiambalá and the Puneña region of Chaschuil (Tinogasta), for which we already had compositional data obtained by other investigators. When discussed within the frame of the interactions between populations of the Puna of Antofagasta de la Sierra and the near valleys the results contribute to the discussion about production and circulation areas of ceramic sets in the past.

Key words: puna and valley interaction - pottery - petrographic characterization - instrumental neutron activation analysis.

Recibido: marzo 2010. Aceptado: diciembre 2012.
Desde momentos tempranos (ca. 9000 años AP), se documenta para la Puna de Antofagasta de La Sierra (en adelante ANS) el registro recurrente de bienes y recursos no locales procedentes de un amplio espacio circumpuneño, que alcanza el área valliserrana meridional, la selva montana y basal, la llanura chaco-santiagueña y la costa pacífica. Los ítems introducidos incluyen un espectro variable, que comprende desde objetos de carácter suntuario o empleados como adorno personal, diversos recursos alimentarios incorporados a la dieta, hasta variadas tecnofacturas y materias primas incorporadas a múltiples fines tecnológicos (Aschero et al. 2002; Babot 2004; Cohen 2005; Escola 2007; Hocsman 2006; Hocsman et al. 2004; López Campeny 2000, 2001, 2009; Olivera 1992; Rodríguez y Martínez 2001, entre otros). La presencia de estos elementos alóctonos en los sitios puneños ha sido interpretada por los autores citados como prueba de la existencia de relaciones a distancia, entabladas entre las poblaciones que habitaron ANS y las que ocuparon diferentes áreas lejanas. Más aún, la recurrencia observada en el registro de los mismos recursos foráneos, a lo largo de una secuencia de casi 10.000 años, permitió plantear esta continuidad como el reflejo de redes sociales de interacción, de posible naturaleza familiar, que se fueron consolidando y reforzando a lo largo del tiempo (Aschero 2006, 2007; López Campeny et al. 2011).

En el caso de los elementos ecofactuales, o bien de tecnofacturas elaboradas sobre ciertos recursos naturales en los cuales la materia prima no ha sufrido grandes modi-

\footnotetext{
1 Instituto Superior de Estudios Sociales (ISES-CONICET) e Instituto de Arqueología y Museo (IAM-UNT). San Martín 1545, San Miguel de Tucumán, CP: 4000, ARGENTINA. Email: marisalopezc@hotmail.com
} 
ficaciones durante el proceso de producción, la identificación de la procedencia ha podido concretarse a través de la aplicación de distintas técnicas de determinación de los materiales y el análisis de las áreas de procedencia naturales que, en conjunto, permitieron establecer, de manera más o menos precisa y directa, una fuente de origen. Sin embargo, otro tipo de bienes -que como veremos con más detalle en el acápite siguiente también han sido interpretados como de carácter alóctono en el registro arqueológico de ANS - no pueden ser englobados en esta categoría anterior, debido principalmente a que una serie de acciones sucesivas, involucradas en su manufactura, suelen alterar las características de la materia prima original, complejizando su vinculación con la fuente de procedencia. Se trata de un conjunto particular de tecnofacturas cerámicas que han sido recuperadas de manera recurrente en los sitios puneños, y cuyo carácter no local ha sido inferido a partir de una perspectiva interpretativa, basada en la identificación de un conjunto de semejanzas tecnológicas y representativas compartidas (o una misma tradición alfarera) con cerámicas recuperadas en otras áreas geográficas, específicamente en los valles meridionales (Babot et al. 2006; Haber 2001, 2006, 2007; Olivera 1992; Olivera y Podestá 1993; Olivera y Vigliani 2000-02; Podestá y Manzi 1995, entre otros). Nos referimos a los estilos cerámicos conocidos bajo los rótulos de Saujil y Ciénaga (González 1977; Sempé 1977, 1993), definidos basados en hallazgos efectuados inicialmente en el valle de Hualfín y de Abaucán, ambos en la provincia de Catamarca. Sin embargo, destacamos que estos planteos se han apoyado exclusivamente, hasta el momento, en una escala de análisis macroscópica, asumiéndose el carácter "alóctono" -léase vallista- de estas cerámicas en los contextos puneños, a partir de las semejanzas formales identificadas con respecto a los estilos definidos inicialmente para los valles. En este sentido, si bien consideramos que la detección de semejanzas estilísticas puede constituir un punto de partida válido para examinar propuestas vinculadas a la circulación de bienes, también creemos que las similitudes formales detectadas no constituyen una prueba en sí misma de la existencia de un origen común para las piezas, pudiendo existir casos de imitaciones o "copias" de estilos por diferentes artesanos, u otras situaciones producto de la dinámica vinculada con el movimiento de personas o poblaciones que llevan consigo sus propias tradiciones cerámicas y el conocimiento tecnológico a otros lugares de producción, solo por mencionar algunos de los complejos factores que podrían estar operando detrás de las semejanzas formales relevadas.

Es por ello que, en el marco de la problemática arqueológica local, consideramos relevante presentar aquí los resultados obtenidos, vinculados con la caracterización composicional petrográfica y química - por corte delgado y análisis de activación neutrónica instrumental (AANI) de un conjunto cerámico recuperado en sitios de la microrregión de ANS. La muestra analizada se encuentra integrada tanto por fragmentos vinculados con los estilos "valliserranos" en discusión (Saujil y Ciénaga), como por ejemplares interpretados como de producción puneña local. Los resultados obtenidos fueron a continuación comparados con los datos de caracterización composicional disponibles para muestras cerámicas -correspondientes a los estilos alfareros valliserranos aludidos- procedentes de sitios arqueológicos del área de valles meridionales (Hualfín), del Bolsón de Fiambalá y de la región puneña de Chaschuil (Tinogasta), que han sido publicados por otros investigadores (Ratto et al. 2002, 2005, 2007; Ratto 2007; Zagorodny et al. 2008) (Figura 1). De lo expuesto anteriormente se desprende que, a partir de los patrones de semejanza o variabilidad composicional detectados entre las muestras comparadas -tanto la de recuperación local como las extra puneñas- además de su vinculación con la geología regional, podremos contrastar, con mayores elementos de prueba, la hipótesis básica hasta ahora sostenida, respecto de la procedencia foránea de estas cerámicas en los sitios puneños; o bien refutar la misma, explorando otras posibles alternativas para explicar este registro arqueológico, pero contando con un cúmulo de nueva evidencia composicional que no se consideró en la discusión previa, restringida, como ya dijimos, a una perspectiva formal macroscópica.

\section{* Antecedentes arqueológicos para un PLANTEO DE LA PROBLEMÁTICA}

El departamento de ANS se localiza en el ángulo noroeste de la provincia de Catamarca, y se encuentra incluido en el denominado sector geográfico Meridional o Austral de la Puna Argentina (ver Figura 1). Su rango altitudinal oscila entre los 3400 a 4200 m.s.n.m. y el paisaje, producto de una intensa actividad tectónica y volcánica 


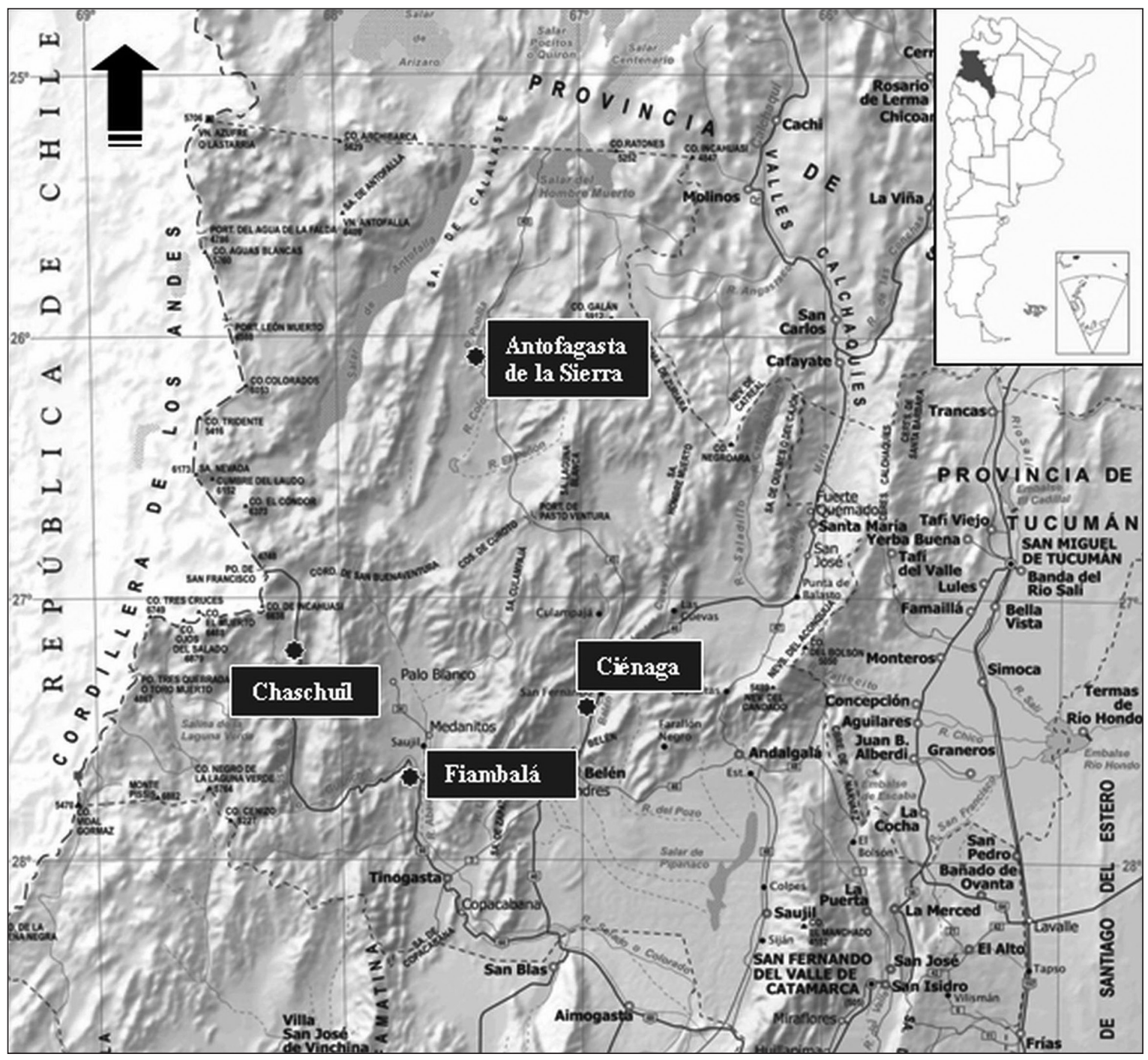

Figura 1. Mapa con la ubicación geográfica de las áreas consideradas en el análisis cerámico composicional.

durante las eras Terciaria y Cuaternaria, se presenta dominado por coladas basálticas, salares y extensas dunas de arena (Valero Garcés et al. 2007). Sin embargo, la presencia de una cuenca endorreica, conformada por la red hidrográfica del río Punilla - de régimen permanente- $y$ dentro de los cuales se destacan los ríos Las Pitas y Miriguaca, da lugar a vegas y ambientes aptos para el desarrollo de actividades pastoriles, la producción de cultivos microtérmicos a pequeña y mediana escala, distintas alternativas de caza y la consecuente posibilidad del desarrollo de poblaciones sedentarias. El perfil altimétrico de estos cauces permanentes permite establecer, en recorridos menores a $20 \mathrm{~km}$, zonas con características diferentes, sobre la base de su topografía y de acuerdo a la disponibilidad de distintos recursos vegetales, faunísticos y minerales (Aschero 2006).

Como hemos anticipado, ejemplares cerámicos que presentan atributos tecnológicos asociados a los estilos valliserranos aludidos han sido recuperados - tanto en superficie como en estratigrafía- en diversos contextos arqueológicos de ANS, incluyendo espacios de carácter funerario, domésticos residenciales y productivos. Las piezas y fragmentos se caracterizan por mostrar 
evidencias de desgaste y frecuentes señales de reparación (Babot et al. 2006; Cohen 2005; López Campeny 2001, 2010; Olivera 1992).

Al respecto de la problemática puntual de interés de este trabajo, podemos decir que los primeros análisis sistemáticos de la cerámica antofagasteña - desde una perspectiva tecnológica- fueron integrados en la tesis doctoral de D. Olivera (1992). Allí, el autor identificó, entre los tipos tecnológicos definidos, un conjunto de fragmentos grises incisos y pulidos en líneas de "filiación valliserrana", asociados al componente ocupacional superior del sitio Casa Chávez Montículos (Olivera 1992: 236). La misma observación se amplía a otros sitios de la microrregión como Casa Chávez Lomas, Casas Viejas A y B, Río Miriguaca 1 y 2, Peñas Chicas, Peñas Coloradas y Real Grande 1 y 4, así como a otros asentamientos del área de Tebenquiche y el salar de Antofalla, entre cuyos materiales Olivera destaca la presencia de “...una gran variedad de tipos cerámicos tempranos relacionados con la Región Valliserrana..." (Olivera 1992: 239). Asimismo, en una publicación posterior, los tipos cerámicos asimilables a los rótulos Ciénaga y Saujil recuperados en sitios de ANS, son aludidos explícitamente como "cerámicas de la Región Valliserrana" (Olivera y Vigliani 2000-02: 465), o "propias de los valles mesotermales" (ibíd.: 477). De manera similar, Babot et al. (2006: 72) concluyen, a partir del análisis de contextos domésticos del sitio Punta de la Peña 9 (I), en la microrregión de ANS, que "Los vínculos con el área valliserrana se manifiestan en elementos de la tecnología cerámica...", a partir de la identificación de conjuntos artefactuales “... asimilables a los típicos de los valles de Abaucán [...] y Hualfín".

Estas conclusiones, sustentadas desde un punto de vista exclusivamente estilístico, se tradujeron en reflexiones con implicancias en la dinámica de las poblaciones locales cuando, por ejemplo, se interpretaron como el reflejo "...de fuertes influencias de los valles mesotermales del Noroeste Argentino..." (Olivera 1992: 236). Así, el incremento registrado en los componentes superiores del sitio Casa Chávez Montículos de los tipos cerámicos que venimos aludiendo se correlacionó con un "...significativo aumento de las relaciones con los valles mesotermales del NOA, en particular Hualfín y Abaucán". (ibídem: 312); e incluso las mencionadas influencias fueron postuladas como resultado de "...un posible avance de los grupos de la región valliserrana hacia los oasis de Puna" (ibídem: 236). Al respecto, la hipó- tesis propuesta por el autor es que a partir de ca. 2000 a 1700 años AP, grupos de poblaciones valliserranas comenzaron a "...ocupar en forma cada vez más intensiva y permanente los 'oasis' de la Puna Meridional" (Olivera 1992: 318). Este proceso habría desembocado en "...un incremento de población en la cuenca y una ocupación más extensiva del espacio regional" (Olivera 1992: 239). Más aún, se propuso que, como resultado de estas "influencias" y/o desplazamientos de los grupos procedentes de los valles mesotermales -contactos inferidos siempre a partir del registro cerámico y sus características estilísticas- se habrían producido cambios en ciertos componentes económicos, a través de un incremento en la incidencia de la actividad agrícola en las comunidades puneñas, caracterizadas por un énfasis en la logística pastoril. Estas modificaciones habrían estado asociadas a cambios en el manejo del espacio productivo, con una mayor optimización en su aprovechamiento (Olivera 1992; Olivera y Podestá 1993; Olivera y Vigliani 2000-02).

Cerámicas asimilables a estos mismos estilos tecnológicos han sido recuperadas en otros sitios puneños, como en la aldea arqueológica de Tebenquiche Chico, emplazada en el salar de Antofalla, donde también han sido inventariadas entre el conjunto de elementos procedentes de los valles meridionales (Granizo 2001; Haber 2001, 2006, 2007; Schuster 2007). Sin embargo, su presencia en los recintos domésticos de este sitio ha sido interpretada desde una perspectiva diferente. A partir de un análisis comparativo de los contextos de asociación, entendidos en términos de "prácticas sociales de participación", Haber (2007) señala diferencias entre los conjuntos cerámicos locales y los foráneos. Estos últimos aparecen asociados a los contextos domésticos, habiendo sido depositados en rellenos de pozos debajo de los pisos, a la vez que fueron excluidos de otros contextos ritualizados, como la fundación de la casa y los eventos funerarios. Sin embargo, señala el autor que estas cerámicas "grises" muestran algunas características remarcables, como una altísima frecuencia de reparación, sugiriendo una preocupación por su conservación, y superficies y bordes de fractura manchados con pigmentos, práctica asociada a la cerámica recuperada en contextos funerarios locales (Krapovickas 1955). Basado en estos atributos, el autor interpreta que la conservación de estas cerámicas vallistas y su particular forma de depósito se habría vinculado con su carácter de "soporte de narrativas de las largas distancias que debieron 
recorrer" con el valor de representar materialmente la "experiencia de vinculación con gente distante" (Haber 2007 : 66); en el marco de interacciones que, durante el primer milenio DC, se han interpretado como caracterizadas por el intercambio a larga distancia de bienes de alto valor agregado, complejidad técnica, sumamente elaborados y social y ritualmente connotados (Haber 2001, 2007).

Pero es Aschero (2007: 100) quien presenta más recientemente una propuesta -que creemos más abarcativapara explicar el hallazgo de estas cerámicas "grises", a las que interpreta como "valliserranas en principio". Para ello parte del hecho, que ya hemos señalado, de su recuperación en los sitios de ANS en asociación a diversos contextos, especialmente los domésticos residenciales, donde aparecen integradas a las diferentes actividades cotidianas. Recordemos que antes señalamos también que la mayoría presenta evidencias de desgaste, lo que podría vincularse con un intenso uso antes de su descarte, e indicios de mantenimiento y conservación, atestiguados por numerosos y frecuentes orificios de reparación. Así, sin descartar la hipótesis de un origen valliserrano original, en términos de los modos tecnológicos de hacer, Aschero propone una lectura a nuestro entender más amplia, cuando sugiere que estas cerámicas pueden interpretarse como el producto artesanal de “...mujeres que vienen de afuera -elegidas como cónyuges en lugares distantes- acompañadas de sus vasijas o de sus particulares conocimientos sobre cómo hacer cerámica. Mujeres que en los espacios domésticos en que operan están dejando las vasijas que traen o produjeron localmente, repitiendo las formas por ellas conocidas" (Aschero 2007: 100).

Recapitulando, podemos concluir que numerosos investigadores - desde diferentes perspectivas teóricas e interpretativas - han coincidido en remarcar las notables similitudes estilísticas relevadas entre ciertos tipos cerámicos recuperados en ANS y otras áreas de la Puna Meridional, con los tipos definidos para sitios de los valles meridionales (especialmente los estilos Ciénaga y Saujil). Estas semejanzas han sido interpretadas como indicadores de vínculos entre los grupos que habitaban ambas áreas geográficas, ${ }^{2}$ plasmados en la presencia de cerá-

2 Los vínculos entre las poblaciones puneñas y las del área valliserrana también se sustentaron en la identificación de relaciones estilísticas entre el arte rupestre local y los estilos cerámicos "va- mica foránea -que se asumió como de origen vallistaobtenida por las poblaciones puneñas, a través de redes de intercambio de productos. Aún más, sobre la base de esta hipótesis se formularon interpretaciones de diversa índole, vinculadas con la dinámica de las poblaciones de ambas áreas en sus más diversos aspectos (demográficos, económicos, rituales, sociales, etc.), pero sustentadas exclusivamente a partir de evidencia macroscópica sin contar, hasta ahora, con datos composicionales que la apoyaran. ${ }^{3}$ Es por ello que consideramos relevante presentar aquí los datos obtenidos a partir de la caracterización petrográfica y química de un conjunto cerámico local, para continuar profundizando en esta problemática, pero partiendo del análisis de nueva evidencia, no disponible hasta ahora.

\section{* Conformación de la muestra cerámica LOCAL: PROCEDENCIA Y COMPOSICIÓN}

Los materiales cerámicos sobre los que se han efectuado los análisis composicionales que aquí presentamos proceden de intervenciones realizadas en dos sitios arqueológicos locales. El primero de ellos es el sitio Punta de la Peña 9 (en adelante $\mathrm{PP}_{9}$ ), que se encuentra emplazado sobre la margen sur del río Las Pitas, a una altitud de unos 3.640 m.s.n.m. El sitio está conformado por una se-

llistos" (Olivera 1992; Olivera y Podestá 1993; Podestá y Manzi 1995). Análisis posteriores integraron, al examen comparativo entre estos dos soportes, la iconografía textil plasmada en prendas recuperadas en sitios puneños (López Campeny 2000, 2001). Más recientemente, Escola y Hocsman (2008) plantearon relaciones a partir de la identificación de un "tipo morfológico recurrente", materializado en un artefacto lítico particular -las raederas de módulo grandísimo (RMG) - que cuenta con ejemplares recuperados en ANS y en sectores valliserranos próximos, como el Valle de Hualfín.

3 De hecho, los análisis tecnológicos de los conjuntos cerámicos de ANS son escasos, pudiendo destacarse los aportes de Vigliani (1999) y Vidal (2002). La primera logró diferenciar categorías generales de uso, a partir de la definición de las capacidades funcionales, o propiedades físico-mecánicas de las pastas, realizando análisis de porosidad (Vigliani 1999). También Vidal (2002) realizó estudios arqueométricos entre los que se destacan análisis para estimar la resistencia mecánica (flexión y compresión) de las piezas cerámicas a la fractura, deformación, abrasión, etc. Sin embargo, ninguno de estos relevantes aportes incluyó análisis composicionales que apuntaran a resolver la problemática de la procedencia de los materiales. 
rie de estructuras arquitectónicas de planta sub-circular y bloques de roca ignimbrita de gran tamaño, dispuestas sobre una extensa planicie fluvial y un sector del talud de derrumbes, al pie de un gran farallón de ignimbritas. La secuencia de ocupación se inicia hace ca. 2.000 años AP (López Campeny 2001, 2010) y se extiende hasta momentos históricos (Cohen 2005), evidenciando un uso recurrente de los espacios para la realización de múltiples actividades. Estas incluyen una variedad de prácticas residenciales - procesamiento, consumo, almacenamiento, uso y descarte de diversas materialidades- una serie de actividades productivas -agrícolas, pastoriles, talla lítica, confección de cuentas, arte rupestre-y contextos asociados a inhumaciones de variadas características. Dicha secuencia cuenta con un total de doce fechados radiocarbónicos (Tabla 1), comprendidos entre 1970 y 380 años AP (Babot et al. 2006; Cohen 2005; López Campeny 2001, 2009, 2010; López Campeny et al. 2005; López Campeny y Escola 2007).

El segundo asentamiento del que proceden los fragmentos cerámicos corresponde al sitio Piedra Horadada 2 (en adelante $\mathrm{PH} 2$ ), también emplazado sobre la margen sur del río Las Pitas, a una altitud de aprox. 3640 m.s.n.m. y a $600 \mathrm{~m}$ al NE de PP9. Constituye un asentamiento a cielo abierto, integrado por estructuras arquitectónicas de variadas dimensiones y planta predominantemente circular, dispersas entre enormes bloques de derrumbes, que se disponen aprovechando la pendiente en el talud del farallón.

Diferentes sectores del sitio han sido interpretados como espacios destinados a uso productivo (corrales), unidades con actividades de residencia-procesamiento-consumo, áreas vinculadas a actividades de talla lítica y espacios de actividad ritual de diferente naturaleza, incluyendo distintas manifestaciones de arte rupestre. La secuencia de ocupación del sitio $\mathrm{PH} 2$, documentada por una serie de seis fechados radiocarbónicos (Tabla 1), se inicia en torno a 1900 años AP y se extiende hasta momentos prehispánicos tardíos, hace aproximadamente unos 600 años AP (López Campeny 2009; López Campeny et al. 2005).

Respecto a la conformación de la muestra cerámica que fue sometida a análisis desde un punto de vista composicional, se siguió un criterio que nos permitiera poner a prueba la hipótesis general hasta ahora postulada, es decir, que el conjunto cerámico recuperado en los sitios puneños, que comparte atributos formales macroscópicos con los estilos definidos para el área valliserrana, es de elaboración no local, y que habría llegado a estos espacios de actividad, desde su lugar de origen en los valles, como resultado de las interacciones - de diversa naturaleza según los autores reseñados- entre las poblaciones que ocuparon ambas áreas. Basados en esta hipótesis, seleccionamos de ambos sitios en estudio una muestra integrada tanto por tiestos vinculados con los estilos "valliserranos", como con ejemplares interpretados como de producción local puneña. El primer grupo (foráneo) fue designado con la letra A y el segundo (local) se identificó con la letra B.

Presentamos a continuación las características tecnológicas macroscópicas presentadas por cada conjunto, mientras que detalles referidos a los análisis efectuados en los tiestos se presentan en los acápites siguientes.

El grupo A (conjunto foráneo) está integrado por fragmentos cerámicos $(\mathrm{N}=18)$ que se seleccionaron después de la identificación de patrones tecnológicos y representativos compartidos con la denominada "porción Ciénaga" de la Colección Muñiz Barreto (Museo de La Plata) y estilos relacionados a ésta, como la denominada cerámica Saujil (González 1977; Sempé 1977, 1993). Un único ejemplar ha podido ser relacionado -desde un aspecto tecnológico y representativo superficial- con el estilo cerámico Aguada. En términos amplios, y desde una perspectiva macroscópica, incluye tiestos de pastas compactas y muy finas, con un reducido porcentaje de antiplástico, de distribución homogénea y tamaño uniforme, generalmente no distinguible a ojo desnudo. Poseen paredes de poco grosor (entre 0,3 a $0,5 \mathrm{~cm}$ ) y superficies tratadas mediante técnicas de pulido, pulido diferencial o en líneas, incisión y grabado, incluyendo frecuentemente la representación de diseños no figurativos y zoomorfos. En general, presentan un alto grado de coherencia, gran resistencia a fracturas y evidencias de una buena cocción, en todos los casos de tipo reductora. No presentan señales de alteración térmica que permitan inferir usos culinarios, aunque algunos ejemplares muestran huellas de reparación y mantenimiento, así como diferentes grados de meteorización y alteración superficial. Con respecto a la morfología de las piezas, es posible decir que la estimación del diámetro de la 


\begin{tabular}{|c|c|c|c|c|c|}
\hline Sigla $^{1}$ & $\begin{array}{l}\text { Sitio y unidad de } \\
\text { Procedencia }\end{array}$ & Muestra datada & Fecha convencional & $\begin{array}{l}\text { Fecha Calibrada }^{2} \\
2 \text { sigmas }(95 \%)\end{array}$ & Referencia bibliográfica \\
\hline $\begin{array}{l}\text { UGA } \\
9076\end{array}$ & $\mathrm{PP}_{9}$ (III) E2 nivel 6 & $\begin{array}{l}\text { Guano de } \\
\text { camélido }\end{array}$ & $1970 \pm 50 \mathrm{AP}$ & $110 \mathrm{AC}$ a $140 \mathrm{cal} \mathrm{DC}$ & López Campeny 2001, 2010 \\
\hline LP 1875 & $\mathrm{PH}_{2} \mathrm{E}_{5}$ nivel 14 & $\begin{array}{l}\text { Guano de } \\
\text { camélido }\end{array}$ & $1870 \pm 100 \mathrm{AP}$ & $60 \mathrm{AC}$ a $410 \mathrm{cal} \mathrm{DC}$ & López Campeny 2009 \\
\hline LP 1716 & $\mathrm{PH}_{2} \mathrm{E}_{5}$ nivel 12 & $\begin{array}{l}\text { Guano de } \\
\text { camélido }\end{array}$ & $1670 \pm 60 \mathrm{AP}$ & 240 a $540 \mathrm{cal} \mathrm{DC}$ & López Campeny 2009 \\
\hline LP 1876 & $\mathrm{PH}_{2} \mathrm{E}_{5}$ nivel 5 & $\begin{array}{l}\text { Guano de } \\
\text { camélido }\end{array}$ & $1630 \pm 90 \mathrm{AP}$ & 220 a $620 \mathrm{cal} \mathrm{DC}$ & López Campeny 2009 \\
\hline $\begin{array}{l}\text { UGA } \\
9069\end{array}$ & $\mathrm{PP}_{9}$ (III) E2 nivel 3 & $\begin{array}{l}\text { Semillas de } \\
\text { chañar }\end{array}$ & $1480 \pm 40 \mathrm{AP}$ & 530 a $660 \mathrm{cal} \mathrm{DC}$ & López Campeny 2001, 2010 \\
\hline LP 1473 & $\begin{array}{l}\mathrm{PP}_{9}(\mathrm{I}) \mathrm{E}_{3} \\
\text { nivel } 2\end{array}$ & Carbón & $1410 \pm 70 \mathrm{AP}$ & 530 a $780 \mathrm{cal} \mathrm{DC}$ & Babot et al. 2006 \\
\hline LP 1430 & $\begin{array}{l}\mathrm{PP}_{9}(\mathrm{I}) \mathrm{E}_{3} \\
\text { nivel } 2\end{array}$ & $\begin{array}{l}\text { Guano de } \\
\text { camélido }\end{array}$ & $1430 \pm 60 \mathrm{AP}$ & 530 a $690 \mathrm{cal} \mathrm{DC}$ & Babot et al. 2006 \\
\hline $\begin{array}{l}\text { UGA } \\
9261\end{array}$ & $\mathrm{PP}_{9}$ (III) $\mathrm{E}_{3}+\mathrm{E}_{4}$ capa 6 & $\begin{array}{l}\text { Guano de } \\
\text { camélido }\end{array}$ & $1340 \pm 50 \mathrm{AP}$ & 600 a $780 \mathrm{cal} \mathrm{DC}$ & Cohen 2005 \\
\hline $\begin{array}{l}\text { UGA } \\
15106\end{array}$ & $\mathrm{PP}_{9}$ (III) $\mathrm{E}_{3}+\mathrm{E}_{4}$ capa $6 \mathrm{D}$ & Hueso humano & $1290 \pm 50 \mathrm{AP}$ & 650 a $870 \mathrm{cal} \mathrm{DC}$ & Cohen 2005 \\
\hline $\begin{array}{l}\text { UGA } \\
15101\end{array}$ & $\begin{array}{l}\mathrm{PP}_{9}(\mathrm{I}) \mathrm{E}_{2} \\
\text { nivel } 4\end{array}$ & Semilla de chañar & $1270 \pm 50 \mathrm{AP}$ & 660 a $880 \mathrm{cal} D C$ & López Campeny y Escola 2007 \\
\hline LP 1887 & $\mathrm{PH}_{2} \mathrm{E}_{2}$ nivel 3 & Carbón & $1270 \pm 50 \mathrm{AP}$ & 660 a $880 \mathrm{cal} \mathrm{DC}$ & López Campeny 2009 \\
\hline $\begin{array}{l}\text { UGA } \\
15104\end{array}$ & $\mathrm{PP}_{9}$ (I) E1 & Hueso humano & $1240 \pm 50 \mathrm{AP}$ & 660 a $890 \mathrm{cal} \mathrm{DC}$ & Babotetal. 2009 \\
\hline $\begin{array}{l}\text { UGA } \\
9070\end{array}$ & $\begin{array}{l}\mathrm{PP}_{9} \text { (III) } \mathrm{E}_{2} \\
\text { nivel } 5\end{array}$ & Carbón & $1150 \pm 150 \mathrm{AP}$ & 600 a $1250 \mathrm{cal} \mathrm{DC}$ & López Campeny 2001, 2010 \\
\hline $\begin{array}{l}\text { UGA } \\
9067\end{array}$ & $\mathrm{PP}_{9}$ (III) E3 capa5 & Carbón & $706 \pm 50 \mathrm{AP}$ & $\begin{array}{c}1210 \text { a } 1130 \mathrm{cal} \text { DC y } 1340 \text { a } 1400 \\
\mathrm{cal} \mathrm{DC}\end{array}$ & Cohen 2005 \\
\hline LP 1572 & $\mathrm{PH}_{2} \mathrm{E}_{1}$ nivel 4 & Carbón & $690 \pm 60 \mathrm{AP}$ & 1220 a $1410 \mathrm{cal} \mathrm{DC}$ & López Campeny et al. 2005 \\
\hline $\begin{array}{l}\text { UGA } \\
9260\end{array}$ & $\begin{array}{l}\mathrm{PP}_{9} \text { (III) } \mathrm{E}_{2} \\
\text { nivel } 2\end{array}$ & Carbón & $600 \pm 50 \mathrm{AP}$ & $1280 \mathrm{a} 1420 \mathrm{cal} \mathrm{DC}$ & López Campeny 2001, 2010 \\
\hline $\operatorname{LP} 1620$ & $\mathrm{PH}_{2} \mathrm{E}_{1}$ nivel 3 & Carbón & $580 \pm 60 \mathrm{AP}$ & 1290 a $1440 \mathrm{cal} \mathrm{DC}$ & López Campeny et al. 2005 \\
\hline LP 1553 & $\begin{array}{l}\mathrm{PP}_{9}(\mathrm{III}) \mathrm{E}_{3} \\
\text { сара } 3\end{array}$ & Carbón & $380 \pm 70 \mathrm{AP}$ & 1420 a $1650 \mathrm{cal} \mathrm{DC}$ & Cohen 2005 \\
\hline
\end{tabular}

Tabla 1. Datos radiométricos vinculados con los dos sitios arqueológicos de Antofagasta de la Sierra de los cuales proceden los materiales cerámicos analizados.

1 Siglas: LP: Laboratorio de Tritio y Radiocarbono (LATYR), Universidad Nacional de La Plata; UGA: Center for Applied Isotope Studies (CAIS), Universidad de Georgia.

2 Las edades radiocarbónicas convencionales fueron calibradas usando el programa Oxcal 3.08. 


\begin{tabular}{|c|c|c|c|c|c|c|c|}
\hline $\mathbf{N}^{\circ}$ & Porción & $\begin{array}{l}\text { Grosor } \\
(\mathrm{cm})\end{array}$ & $\emptyset$ Boca $(\mathrm{cm})$ & $\begin{array}{l}\text { Pasta y } \\
\text { cocción }^{1}\end{array}$ & $\begin{array}{c}\text { Tratamiento } \\
\text { Externo/Interno }\end{array}$ & Estilo cerámico & Morfología \\
\hline$\underline{62}$ & Borde & 0,50 & 22 & $\mathrm{C} / \mathrm{R}$ & Pul.- inc./Pulido & Ciénaga & Puco \\
\hline$\underline{47}$ & Borde & 0,35 & 18 & $\mathrm{C} / \mathrm{R}$ & Pul.-grab./Pulido & Ciénaga & Puco \\
\hline$\underline{48}$ & Borde & 0,30 & 20 & $\mathrm{C} / \mathrm{R}$ & Pul.- inc./Pulido & Ciénaga & Puco \\
\hline$\underline{74}$ & Borde & 0,35 & 23 & $\mathrm{C} / \mathrm{R}$ & Pul.-grab./Pul. Lin. & Ciénaga & Vaso- Jarro \\
\hline 90 & Cuerpo & 0,50 & - & $\mathrm{C} / \mathrm{R}$ & N.d./Pulido & N.d. & N.d. \\
\hline$\underline{91}$ & Cuerpo & 0,30 & - & $\mathrm{C} / \mathrm{R}$ & Pulido/Alisado & Ciénaga & N.d. \\
\hline 130 & Borde & 0,20 & 10 & $\mathrm{C} / \mathrm{R}$ & Pul.- inc./Alisado & Saujil & Vaso- Jarra \\
\hline 194 & Cuerpo & 0,45 & - & $\mathrm{C} / \mathrm{R}$ & Pul.-inc./Pul. Lin. & Ciénaga & N.d. \\
\hline 255 & Cuerpo & 0,60 & - & $\mathrm{C} / \mathrm{R}$ & Pulido líneas/N.d. & Ciénaga & N. d. \\
\hline 264 & Cuerpo & 0,50 & - & $\mathrm{C} / \mathrm{R}$ & Pul.-grab./Pulido & Ciénaga & N.d. \\
\hline$\underline{03}$ & Borde & 0,50 & 18 & $\mathrm{C} / \mathrm{R}$ & Pulido/Pulido & N.d. & Puco \\
\hline $4 / 1$ & Cuerpo & 0,45 & - & $\mathrm{C} / \mathrm{R}$ & Pul.- inc./Pulido & Ciénaga & Puco \\
\hline$\underline{4 / 2}$ & Cuerpo & 0,45 & - & $\mathrm{C} / \mathrm{R}$ & Pul.-grab./Pulido & Ciénaga & Puco \\
\hline$\underline{14}$ & Cuerpo & 0,40 & - & $\mathrm{C} / \mathrm{R}$ & Pul.-grab./Pulido & Ciénaga & N.d. \\
\hline$\underline{24}$ & Borde & 0,40 & 11 & $\mathrm{C} / \mathrm{R}$ & Pul.-grab./Pulido & Aguada? & Puco \\
\hline$\underline{27}$ & Cuerpo & 0,40 & - & $\mathrm{C} / \mathrm{R}$ & N.d./ Pulido líneas & Saujil & N.d. \\
\hline 43 & Cuerpo & 0,30 & - & $\mathrm{C} / \mathrm{R}$ & Pul.-grab./Pulido & Ciénaga & N.d. \\
\hline$\underline{46}$ & Cuerpo & 0,40 & - & $\mathrm{C} / \mathrm{R}$ & Pul.-grab./Pulido & Ciénaga & Puco \\
\hline
\end{tabular}

Tabla 2. Tiestos sometidos a corte delgado que conforman el Grupo A. Los números subrayados indican las muestras que además fueron irradiadas (AANI).

\footnotetext{
C/R: compacta y reductora.
}

boca (en el caso de los fragmentos de bordes), sumada al grosor de las paredes y la presencia de algunos puntos característicos en el perfil del ejemplar, permitieron reconocer, entre este grupo, principalmente piezas de tamaño mediano y pequeño, correspondientes morfológicamente a vasos y pucos (Tabla 2, Figuras 2, 3 y 4).

El grupo B (elaboración local) se conformó poniendo especial énfasis en la inclusión de ejemplares correspondientes a los tipos tecnológicos presentes de manera mayoritaria en los sitios puneños y que, además, mostraran claras evidencias de uso doméstico. Está integrado por un conjunto más reducido de fragmentos cerámicos $(\mathrm{N}=9)$ y macroscópicamente se trata de tiestos de pastas porosas, de relativamente baja compactación, en algunos casos muy disgregables al tacto. Predomina la cocción oxidante o bien una cocción de tipo incompleta. Presentan antiplástico de tamaño variable, pero principalmente grueso a muy grueso, siendo claramente visibles a ojo desnudo. Poseen paredes de mayor grosor que los ejemplares del grupo A (entre 0,5 a $0,8 \mathrm{~cm}$ ) y superficies principalmente irregulares, sin tratamiento $y$, en menor grado, alisadas. A diferencia del primer conjunto, algunos de estos tiestos muestran evidencias de exposición al fuego. En este 


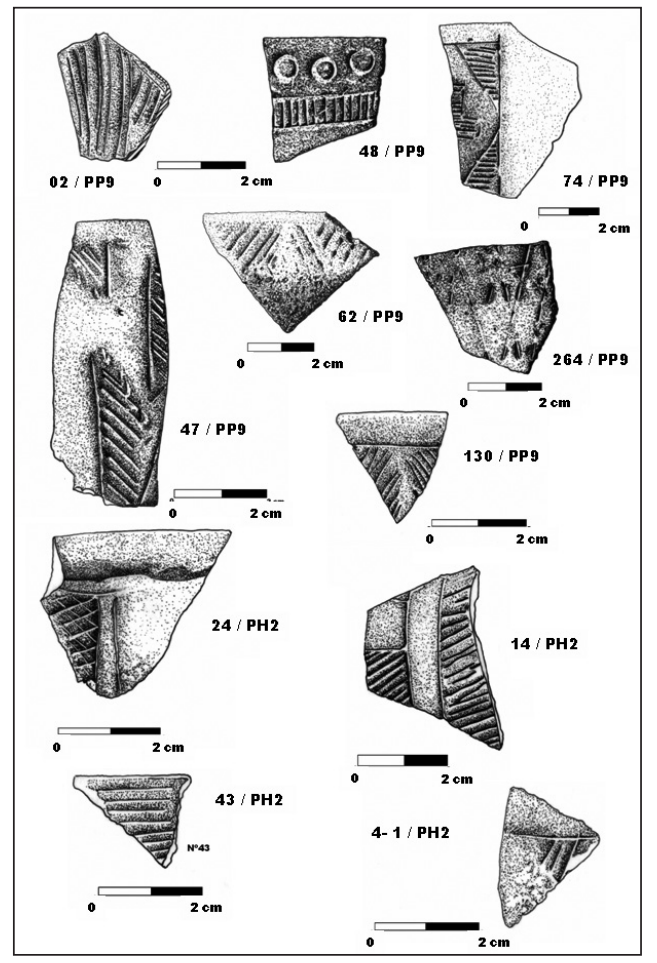

Figura 2. Algunos de los fragmentos cerámicos sometidos a los análisis composicionales que conforman el grupo A, procedentes de los sitios de Antofagasta de la Sierra.

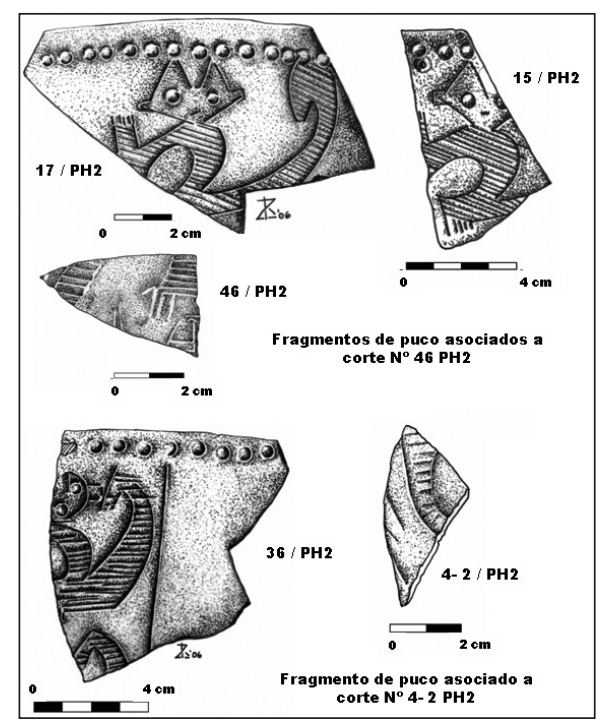

Figura 3. Algunos de los tiestos sometidos a los análisis composicionales que conforman el grupo A, procedentes de los sitios de Antofagasta de la Sierra y su vinculación con fragmentos de piezas mayores.

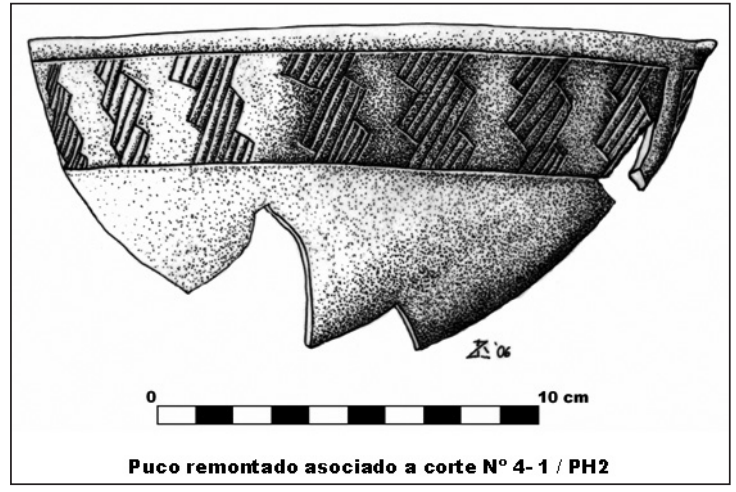

Figura 4. Puco remontado, uno de cuyos fragmentos se sometió a análisis de corte delgado y activación neutrónica instrumental.

caso, debido que la mayoría de los ejemplares corresponden a porciones de cuerpo y carecen de puntos característicos del perfil, es más difícil estimar las morfologías y las dimensiones principales a las que se asociarían estos fragmentos. Sin embargo, podemos decir que es posible inferir la presencia de vasijas de portes mayores que en el grupo A, en el caso de los ejemplares que presentan paredes notablemente más gruesas (Tabla 3 ).

Si partimos de la hipótesis general enunciada, podemos decir que las implicancias principales para su contrastación son las siguientes: en primer lugar, cada uno de los grupos (A y B) debe mostrar una relativa homogeneidad y coherencia interna desde el punto de vista composicional, es decir, que las similitudes observadas a nivel macroscópico deben ser comparables desde el punto de vista microscópico, mostrando los fragmentos de un mismo grupo, un perfil petrográfico y químico afín. En segundo término, los ejemplares pertenecientes a cada uno de los dos grupos de recuperación local deben diferir entre ellos, en relación a las variables composicionales analizadas, es decir, que las diferencias observadas en las piezas superficialmente, deben correlacionarse también con pastas que presenten diferentes componentes identificables a nivel de su composición. Una tercera implicancia a señalar, ya desde un plano comparativo extra-local, es que los ejemplares correspondientes a nuestro grupo A deben ser similares, composicionalmente hablando, a los fragmentos cerámicos equivalentes macroscópicamente (asignados a un mismo estilo), recuperados en los sitios de valles, mientras que los del grupo B deben mostrar pastas que, desde una 
escala microscópica mineral y elemental, se diferencien de éstos últimos. Finalmente, los elementos componentes de la pasta, entre los ejemplares del grupo A, deben poder correlacionarse con los componentes líticos y minerales que caracterizan a los depósitos geológicos de la propuesta como área de origen, es decir, los valles meridionales, a la vez que deberían mostrar diferencias en su composición con los basamentos característicos del área puneña. De modo complementario, los ejemplares que conforman nuestro grupo "local" o B deben mostrar un perfil petrográfico afín con la geología de ANS, a la vez que ciertos elementos de las pastas deberían permitirnos trazar diferencias con la composición de los depósitos de valles. Es importante destacar que estas dos últimas series de afirmaciones implican contar con una base de información independiente de la que puede desprenderse de las muestras en estudio, que no siempre es accesible o está disponible para su consulta. Retomaremos este aspecto luego de exponer los resultados obtenidos, y ahora presentamos a continuación los aspectos metodológicos vinculados con los análisis efectuados en los conjuntos cerámicos.

\section{* Consideraciones metodológicas sobre LOS ANÁLISIS EFECTUADOS}

Una pieza cerámica es el resultado de una sumatoria de decisiones y prácticas, involucradas en una particular "manera de hacer" durante todo el proceso productivo, y solo una parte de estas acciones puedan dejar huellas o trazas observables en este tipo de registro (Cremonte 1996). En este sentido, es importante no perder de vista que la relación entre el artefacto terminado y la fuente de procedencia de la materia prima no es tan directa como en el caso de otras materialidades (por ejemplo, los materiales líticos), por un conjunto de particularidades relacionadas con el proceso productivo de la alfarería (Schuster 2007). Nos referimos al hecho de que durante la elaboración cerámica se suelen alterar las características de las fuentes originales mediante procesos de tamizado, limpieza o selección del material, mezcla de materias arcillosas procedentes de diferentes fuentes o la adición de nuevos elementos como antiplásticos o inclusiones intencionales. Por ello, la aplicación complementaria de diferentes técnicas analíticas para la caracterización de los materiales cerámicos puede aportar notablemente en las interpretaciones de los resultados (Sanhueza et al. 2004).

\section{La técnica de análisis por corte delgado}

Como adelantamos, aplicamos métodos petrográficos para la caracterización composicional de las pastas cerámicas, mediante la descripción de cortes delgados, analizados con microscopio de polarización. ${ }^{4}$ Si bien es un método de bajo costo, se trata de una técnica destructiva; en la cual la preparación y montaje de las láminas, así como su posterior análisis, conllevan demasiado tiempo. Esto determina que siempre deba seleccionarse una muestra cerámica representativa, acorde con la problemática de la investigación. En nuestro caso, el propósito principal fue el de aportar elementos sobre la base de los cuales discutir aspectos relacionados con las posibles áreas generales de procedencia (local y/o foránea) asociadas a la producción de un conjunto particular de piezas cerámicas recuperadas en Antofagasta de la Sierra, partiendo de la hipótesis básica que se ha sostenido hasta ahora, es decir, su origen no local y su procedencia asociada al área de valles meridionales. A partir de los resultados obtenidos, podremos sostener dicha hipótesis, o bien refutarla y proponer interpretaciones respecto de las modalidades adoptadas por las relaciones de interacción, entre comunidades de Puna y de valles, en este caso, vistas a través del análisis composicional mineral de estas tecnofacturas particulares.

Partiendo de este objetivo, el análisis petrográfico microscópico se focalizó en el registro y/o la estimación de los siguientes atributos $\mathrm{y} / \mathrm{o}$ variables:

Matriz. Corresponde a la porción de grano más fina, compuesta por las arcillas y sus componentes minerales naturales. Para su descripción se consideraron aspectos vinculados con la textura, color, porcentaje de porosidad, regularidad de forma, regularidad de orientación e identificación mineral.

Inclusiones. Integradas por los agregados de mayor tamaño relativo. En el caso de las inclusiones se consideró: su

\footnotetext{
4 Las descripciones de los cortes fueron efectuadas por los Drs. F. Sardi y M. Báez, Instituto Superior de Correlación Geológica (INSUGEO), Facultad de Ciencias Naturales e Instituto Miguel Lillo, Universidad Nacional de Tucumán.

5 La ficha de registro empleada para las descripciones fue proporcionada por la Lic. Nora Zagorodny y corresponde a la elaborada en el Laboratorio de Análisis Cerámico, Facultad de Ciencias Naturales y Museo, Universidad Nacional de La Plata.
} 


\begin{tabular}{|c|c|c|c|c|c|c|c|c|}
\hline $\mathbf{N}^{\circ}$ & Porción & $\begin{array}{l}\text { Grosor } \\
(\mathrm{cm})\end{array}$ & $\begin{array}{l}\emptyset \text { Boca } \\
(\mathrm{cm})\end{array}$ & $\begin{array}{l}\text { Pastay } \\
\text { cocción }^{1}\end{array}$ & $\begin{array}{c}\text { Tratamiento } \\
\text { Externo }\end{array}$ & $\begin{array}{c}\text { Tratamiento } \\
\text { Interno }\end{array}$ & Restos & Morfología \\
\hline$\underline{02}$ & Cuerpo & 0,40 & - & $\mathrm{C} / \mathrm{O}$ & Alisado e inciso & Alisado & - & N.d. \\
\hline$\underline{49}$ & Cuerpo & 0,85 & - & $\mathrm{C} / \mathrm{O}$ & Alisado & Alisado & - & N.d. \\
\hline 72 & Cuerpo & 0,55 & - & $\mathrm{P} / \mathrm{O}$ & Alisado & Alisado & Hollín & N. d. \\
\hline$\underline{89}$ & Borde & 0,80 & 30 & $\mathrm{P} / \mathrm{O}$ & Alisado & Alisado & - & Olla- Urna \\
\hline 135 & Cuerpo & 0,75 & - & $\mathrm{P} / \mathrm{R}$ & N.d. & Alisado y engobado & - & N.d. \\
\hline 211 & Cuerpo & 0,75 & - & $\mathrm{C} / \mathrm{RO}$ & Pulido & N. d. & Hollín & N. d. \\
\hline$\underline{38}$ & Cuerpo & 0,80 & - & $\mathrm{P} / \mathrm{O}$ & Alisado & Alisado & Ceniza & N. d. \\
\hline$\underline{76}$ & Borde & 0,80 & n. d. & $\mathrm{P} / \mathrm{R}$ & Alisado & Alisado & Hollín & N. d. \\
\hline$\underline{79}$ & Borde & 0,80 & 23 & $\mathrm{P} / \mathrm{R}$ & Alisado & Alisado & Hollín & N. d. \\
\hline
\end{tabular}

Tabla 3. Tiestos sometidos a corte delgado que conforman el Grupo B. Los números subrayados indican las muestras que además fueron irradiadas (AANI).

1 C/O: compacta y oxidante; $\mathrm{P} / \mathrm{O}$ : porosa y oxidante; $\mathrm{P} / \mathrm{R}$ : porosa y reductora; $\mathrm{C} / \mathrm{RO}$ : compacta redox

porcentaje relativo en relación a la matriz, la orientación de los agregados, el tipo y forma de las inclusiones, su grado de selección, así como la identificación mineral de cristaloclastos y litoclastos, tanto de especímenes predominantes como de ejemplares aislados $y /$ o singulares.

\section{Los análisis de activación neutrónica instrumental}

Además de los análisis de corte delgado, se efectuó la caracterización multielemental por análisis de activación neutrónica instrumental (AANI) a una porción de la muestra cerámica de ANS. Esto nos permitió contar con datos complementarios a los obtenidos a través de la caracterización petrográfica, permitiendo acceder, de este modo, a información contenida en las arcillas o matriz de los tiestos.

La caracterización multielemental por análisis de activación neutrónica instrumental (AANI), apunta a la determinación de la concentración de los elementos químicos presentes en determinados bancos de arcilla e inclusiones minerales utilizados como materia prima para la manufactura de objetos cerámicos. Las relaciones de abundancia de los elementos químicos minoritarios y de los elementos traza presentes deben reflejar las diferencias entre los ambientes geológicos de origen. En el caso espe- cífico de las materias primas utilizadas en la producción alfarera, la composición química de las fuentes arcillosas está determinada por las rocas de origen. De este modo, debido a que la composición química de la pasta de los tiestos depende principalmente de las características de las arcillas empleadas para su manufactura, es posible clasificar las muestras cerámicas en grupos estadísticamente diferenciables entre sí, a partir de las relaciones de abundancia observadas entre los elementos traza y minoritarios (Ratto et al. 2002). De este modo, y de forma complementaria a los análisis de corte delgado -usados para la caracterización composicional petrográfica de cerámica- los AANI permiten acceder, además, a información contenida en las arcillas o matriz de los tiestos. Así, con el empleo de la técnica nuclear es posible identificar los depósitos arcillosos explotados para la producción alfarera, ubicar centros de producción y delinear posibles circuitos de producción y circulación (Ratto 2007).

Sin embargo, a fin de contrarrestar algunas de las principales limitaciones que ofrece esta técnica analítica, es de vital importancia conocer la composición mineralógica de la muestra cerámica sometida a AANI, ya que las inclusiones pueden estar presentes en la composición original de la arcilla, o bien pueden ser agregadas durante el proceso de manufactura. Esto determina que la 
interpretación de los resultados químicos no deba ser nunca lineal ni directa, dado que las relaciones originales de concentración entre varios elementos presentes en la arcilla pueden ser alteradas por procesos tecnológicos de manufactura, fundamentalmente, el agregado de antiplásticos (Ratto 2007; Ratto et al. 2002).

Tratamiento estadístico de los datos. En líneas generales, se siguió la metodología reseñada por Ratto et al. (2002, 2005, 2007). Así, aunque a través del AANI se determinaron 20 elementos conformados por Tierras Raras (Ce, $\mathrm{Eu}, \mathrm{La}, \mathrm{Lu}, \mathrm{Sm}, \mathrm{Tb}, \mathrm{Yb}$ ), Actínidos (Th, U); Alcalinos (Ba, $\mathrm{Cs}, \mathrm{Rb}$ ), Metales de Transición (Co, Cr, Hf, Fe, Sb, Sc y $\mathrm{Ta}$ ) y No metales (As); para el análisis estadístico se seleccionaron solo aquellos: a) cuyas mediciones tuvieran un error menor del 10\%; b) no contaran con datos faltantes o por debajo del límite de detección; y c) se contara con información suficiente sobre su comportamiento en la literatura especializada. De este modo, siguiendo estos criterios se eliminaron para el análisis de componentes básicos los elementos As, Sb y U (cfr. Ratto et al. 2002).

Los datos analíticos, previamente validados de acuerdo a los criterios antes enumerados, fueron sometidos a un análisis numérico multivariado por Componentes Principales (ACP), utilizando el programa estadístico SPSS versión 15.0, con transformación logarítmica de los datos, cálculo de matriz de covarianza y rotación Varimax. Sobre los componentes o factores obtenidos se realizó un Análisis de Conglomerado Jerárquico (ACJ), utilizando el método de vinculación Ward y la Distancia Euclídea al cuadrado, para definir grupos en función de los valores que adquiere cada componente en cada muestra en particular, los que fueron representados en el correspondiente dendograma (Figura 5). Estos grupos fueron luego corregidos mediante Análisis Discriminante (AD), empleando distancia de Mahalanobis. El AD corrige la asignación de los grupos y genera nuevos grupos predictivos o grupos de pertenencia pronosticados, calculando además la probabilidad de pertenencia de cada muestra a un grupo determinado (Ratto et al. 2002, 2005, 2007). En términos sencillos, podemos decir que estas técnicas estadísticas multivariadas se aplican con la finalidad de dividir un conjunto de elementos o variables -en este caso tiestos - en grupos de forma que, por un lado, los perfiles de los elementos que conforman un mismo grupo -aquí se trata específicamente de su composición química multielemental- sean muy similares entre sí, es decir, que el grupo esté cohesionado internamente y, por el otro, los elementos pertenecientes a grupos diferentes tengan un comportamiento distinto con respecto a las variables analizadas, es decir, que cada cual esté aislado externamente de los demás grupos.

\section{* Resultados del anÁlisis composicional PETROGRÁFICO POR CORTE DELGADO}

\section{Determinaciones obtenidas para la muestra cerámica local}

Atributos de la matriz. Las características asociadas a la matriz de los tiestos se encuentran sintetizadas en las Tablas 4 y 5 . Al respecto, podemos decir que en ambos conjuntos cerámicos domina ampliamente una textura arcillosa, de aspecto homogéneo, de grano fino a muy fino. Esta matriz arcillosa puede además ser descrita como laminar en idénticas proporciones en cada uno de los dos grupos. Como rasgos menos frecuentes podemos señalar una textura grumosa en un ejemplar del grupo A y dos tiestos del grupo $\mathrm{B}$ que presentan una textura arcillosa-porosa.

En cuanto al color, es uniforme en todos los casos, sin embargo, puede marcarse una diferencia entre los ejemplares de ambos grupos, en lo que respecta a este atributo. Los fragmentos del grupo A presentan, con excepción de un único caso, un color que varía entre negro o pardo oscuro, a un pardo levemente grisáceo, que permite inferir la presencia de caolinita y montmorillonita entre los minerales arcillosos. Los tiestos del grupo B, en cambio, exhiben una matriz de coloración rojiza uniforme, que se interpretó en relación con la presencia de material ferruginoso en estado oxidado.

Con respecto a la porosidad de la matriz, ambos grupos muestran tendencias similares, con una más elevada proporción de porcentajes bajos, contando con un único caso en el que no pudo determinarse por problemas vinculados con el montaje, y no se han registrado casos de porosidad elevada en ningún ejemplar.

La orientación de las partículas componentes de la matriz permite trazar diferencias entre ambos grupos. Mientras que la mayor proporción de los tiestos pertenecientes al 


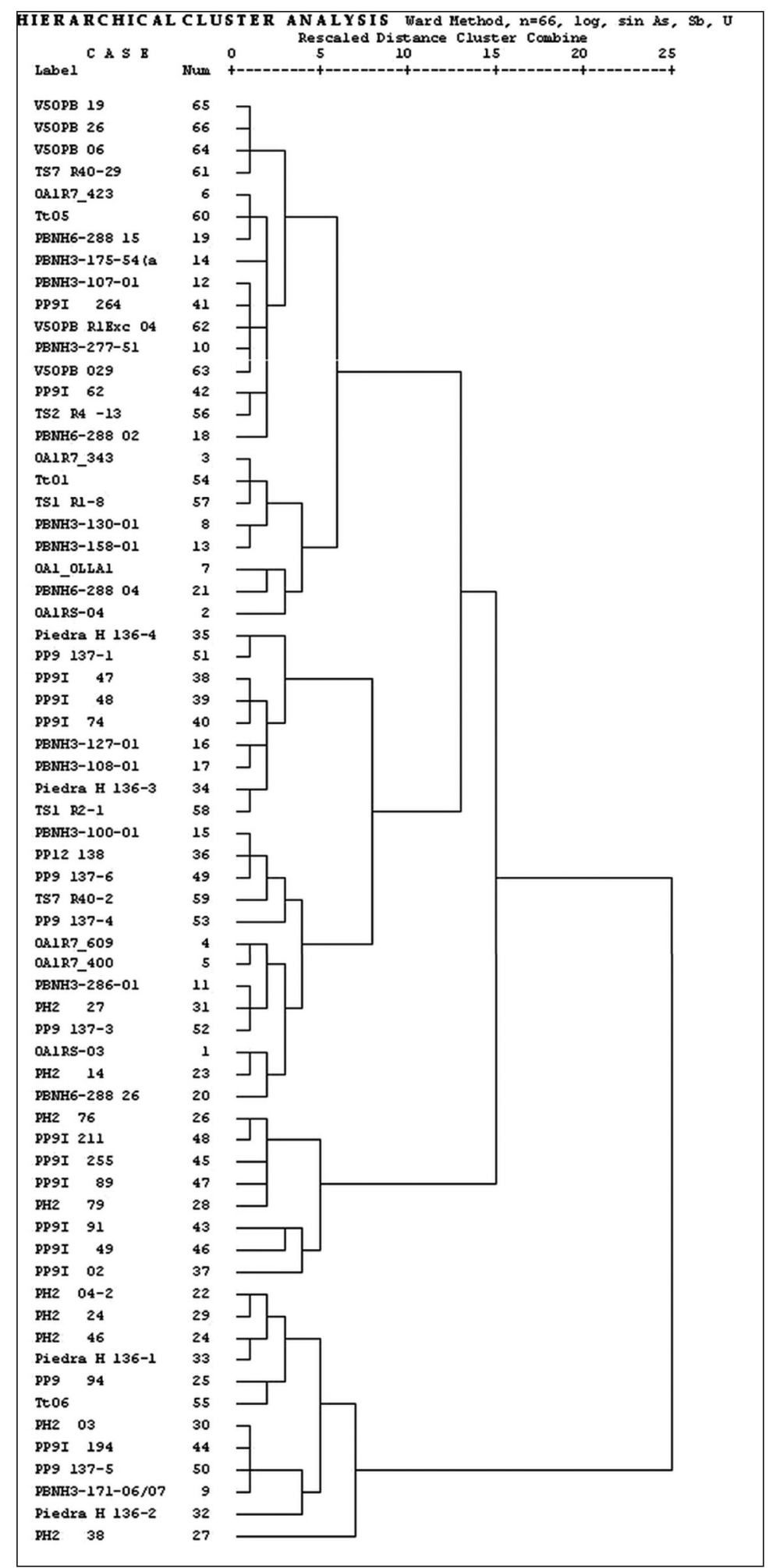

Figura 5. Dendograma que representa los resultados del Análisis de Conglomerado Jerárquico (ACJ) de la muestra sometida a AANI. 


\begin{tabular}{|c|c|c|c|c|c|c|c|c|c|c|c|c|c|c|c|c|c|c|c|}
\hline \multirow{2}{*}{$\mathbf{N}^{\circ}$} & \multicolumn{4}{|c|}{ TEXTURA } & \multicolumn{2}{|c|}{ COLOR } & \multicolumn{3}{|c|}{ \%POROSIDAD } & \multicolumn{2}{|c|}{ FORMA } & \multicolumn{3}{|c|}{ ORIENTACION } & \multicolumn{5}{|c|}{ MINERALES } \\
\hline & $\mathrm{Ar}$ & $\mathrm{La}$ & Po & Gr & Un & $\mathrm{N} / \mathrm{u}$ & $<5$ & $5-15$ & $>15$ & Ir & $\operatorname{Re}$ & $\mathrm{S} / \mathrm{o}$ & $\mathrm{R} / \mathrm{p}$ & $R / t$ & $\mathrm{Ar}$ & Mi & $\mathrm{Fe}$ & Q & $\mathrm{Ca}$ \\
\hline 62 & $\mathrm{x}$ & & & & $\mathrm{x}$ & & & $\mathrm{X}$ & & & $\mathrm{X}$ & $\mathrm{X}$ & & & $\mathrm{X}$ & & & & \\
\hline 47 & $\mathrm{X}$ & $\mathrm{X}$ & & & $\mathrm{X}$ & & $\mathrm{X}$ & & & $\mathrm{X}$ & & & $\mathrm{X}$ & & $\mathrm{X}$ & $\mathrm{X}$ & & & \\
\hline 48 & $\mathrm{X}$ & & & & $\mathrm{X}$ & & & $\mathrm{X}$ & & & $\mathrm{X}$ & & $\mathrm{X}$ & & $\mathrm{X}$ & & & & \\
\hline 74 & $\mathrm{x}$ & & & & $\mathrm{x}$ & & $\mathrm{x}$ & & & $\mathrm{x}$ & & & $\mathrm{x}$ & & $\mathrm{X}$ & & & & \\
\hline 90 & $\mathrm{x}$ & & & & $\mathrm{X}$ & & $\mathrm{x}$ & & & $\mathrm{x}$ & & & & $\mathrm{X}$ & $\mathrm{X}$ & $\chi$ & & & \\
\hline 91 & $\mathrm{X}$ & & & & $\mathrm{X}$ & & $\mathrm{X}$ & & & $\mathrm{X}$ & & $\mathrm{X}$ & & & $\mathrm{X}$ & & & & \\
\hline 130 & $\mathrm{X}$ & & & & $\mathrm{X}$ & & $\mathrm{X}$ & & & & $\mathrm{X}$ & $\mathrm{X}$ & & & $\mathrm{X}$ & & & & \\
\hline 194 & $\mathrm{x}$ & & & & $\mathrm{x}$ & & $\mathrm{x}$ & & & $\mathrm{x}$ & & & $\mathrm{x}$ & & $\mathrm{X}$ & $\chi$ & & & \\
\hline 255 & & & & $\mathrm{X}$ & $\mathrm{X}$ & & & $\mathrm{X}$ & & & & & & & $\mathrm{X}$ & $\chi$ & $\mathrm{X}$ & $\mathrm{X}$ & $\mathrm{X}$ \\
\hline 264 & $\mathrm{X}$ & & & & $\mathrm{X}$ & & $\mathrm{X}$ & & & $\mathrm{X}$ & & & $\mathrm{x}$ & & $\mathrm{X}$ & $\mathrm{X}$ & & & \\
\hline 3 & $\mathrm{X}$ & & & & $\mathrm{X}$ & & $\mathrm{X}$ & & & $\mathrm{X}$ & & & $\mathrm{x}$ & & $\mathrm{X}$ & $\mathrm{X}$ & & & \\
\hline $4 / 1$ & $\mathrm{X}$ & $\mathrm{X}$ & & & $\mathrm{X}$ & & & $\mathrm{x}$ & & & $\mathrm{X}$ & & & $\mathrm{x}$ & $\mathrm{X}$ & $\mathrm{X}$ & & & \\
\hline $4 / 2$ & $\mathrm{x}$ & $\mathrm{X}$ & & & $\mathrm{X}$ & & $x$ & & & & $X$ & & & $X$ & $X$ & $X$ & & & \\
\hline 14 & $x$ & & & & $X$ & & $x$ & & & & & & $\mathrm{X}$ & & $X$ & $\mathrm{X}$ & & & \\
\hline 24 & $\mathrm{X}$ & & & & $X$ & & $\mathrm{X}$ & & & & $X$ & & $\mathrm{X}$ & & $X$ & $\mathrm{X}$ & & & \\
\hline 27 & $\mathrm{X}$ & & & & $X$ & & $\mathrm{X}$ & & & & $\mathrm{X}$ & & $\mathrm{X}$ & & $X$ & $\mathrm{X}$ & & & \\
\hline 43 & $\mathrm{X}$ & & & & $\mathrm{X}$ & & $\mathrm{X}$ & & & $\mathrm{X}$ & & & $\mathrm{X}$ & & $X$ & $\chi$ & & & \\
\hline 46 & $X$ & $X$ & & & $X$ & & & $X$ & & $\mathrm{X}$ & & & & $\mathrm{X}$ & $X$ & $X$ & & & \\
\hline
\end{tabular}

Tabla 4. Atributos de la matriz en tiestos del grupo A. Referencias: Textura: Ar: arcillosa; La: laminar; Po: porosa y Gr: grumosa. Color: Un: uniforme y N/u: no uniforme. Forma: Ir: irregular y Re: regular. Orientación: S/o: sin orientación; R/p: regular parcial y R/t: regular total. Minerales: Ar: arcillas; Mi: micas; $(X)$ biotita y $(\chi)$ biotita y muscovita; Fe: minerales de hierro; Q: cuarzo y Ca: calcita.

\begin{tabular}{|c|c|c|c|c|c|c|c|c|c|c|c|c|c|c|c|c|c|c|c|}
\hline \multirow{2}{*}{$\mathbf{N}^{\circ}$} & \multicolumn{4}{|c|}{ TEXTURA } & \multicolumn{2}{|c|}{ COLOR } & \multicolumn{3}{|c|}{ \%POROSIDAD } & \multicolumn{2}{|c|}{ FORMA } & \multicolumn{3}{|c|}{ ORIENTACION } & \multicolumn{5}{|c|}{ MINERALES } \\
\hline & $\mathrm{Ar}$ & $\mathrm{La}$ & Po & $\mathrm{Gr}$ & Un & $\mathrm{N} / \mathrm{u}$ & $<5$ & $5-15$ & $>15$ & Ir & $\operatorname{Re}$ & $\mathrm{S} / \mathrm{o}$ & $\mathrm{R} / \mathrm{p}$ & $\mathrm{R} / \mathrm{t}$ & $\mathrm{Ar}$ & $\mathrm{Mi}$ & $\mathrm{Fe}$ & Q & $\mathrm{Ca}$ \\
\hline 2 & $\mathrm{X}$ & & & & $\mathrm{X}$ & & $\mathrm{X}$ & & & $\mathrm{x}$ & & $\mathrm{x}$ & & & $\mathrm{x}$ & $\mathrm{x}$ & $\mathrm{X}$ & & \\
\hline 49 & $\mathrm{X}$ & & & & $\mathrm{X}$ & & $\mathrm{X}$ & & & $\mathrm{X}$ & & $\mathrm{x}$ & & & $\mathrm{X}$ & & & $\mathrm{X}$ & \\
\hline 72 & $\mathrm{X}$ & $\mathrm{X}$ & & & $\mathrm{X}$ & & $\mathrm{X}$ & & & $\mathrm{X}$ & & $\mathrm{x}$ & & & $\mathrm{X}$ & & $\mathrm{X}$ & & \\
\hline 89 & $\mathrm{X}$ & & & & $\mathrm{X}$ & & & $\mathrm{X}$ & & $\mathrm{X}$ & & $\mathrm{X}$ & & & $\mathrm{X}$ & & $\mathrm{X}$ & & \\
\hline 135 & $\mathrm{X}$ & & $\mathrm{X}$ & & $\mathrm{X}$ & & & & & & & & & & & & & & \\
\hline 211 & $\mathrm{X}$ & & & & $\mathrm{X}$ & & $\mathrm{X}$ & & & & $\mathrm{X}$ & $\mathrm{x}$ & & & $\mathrm{X}$ & $\mathrm{X}$ & $\mathrm{X}$ & & \\
\hline 38 & $\mathrm{X}$ & $\mathrm{X}$ & & & $\mathrm{X}$ & & $\mathrm{X}$ & & & $\mathrm{X}$ & & $\mathrm{x}$ & & & $\mathrm{X}$ & $\mathrm{X}$ & $\mathrm{X}$ & & \\
\hline 76 & $\mathrm{X}$ & & $\mathrm{X}$ & & $\mathrm{X}$ & & & $\mathrm{X}$ & & $\mathrm{X}$ & & $\mathrm{X}$ & & & $\mathrm{X}$ & & $\mathrm{X}$ & & \\
\hline 79 & $\mathrm{X}$ & & & & $\mathrm{X}$ & & $\mathrm{X}$ & & & $\mathrm{X}$ & & $\mathrm{x}$ & & & $\mathrm{X}$ & $\chi$ & $\mathrm{X}$ & & \\
\hline
\end{tabular}

Tabla 5. Atributos de la matriz en tiestos del grupo A. Referencias: Textura: Ar: arcillosa; La: laminar; Po: porosa y Gr: grumosa. Color: Un: uniforme y N/u: no uniforme. Forma: Ir: irregular y Re: regular. Orientación: S/o: sin orientación; R/p: regular parcial y R/t: regular total. Minerales: Ar: arcillas; Mi: micas; $(X)$ biotita y $(\chi)$ biotita y muscovita; Fe: minerales de hierro; Q: cuarzo y Ca: calcita. 
grupo A exhiben una orientación parcial o total de las inclusiones -fundamentalmente de los minerales micáceos- los ejemplares del grupo B se caracterizan, en todos los casos en que pudo determinarse, por la ausencia de orientación de los minerales componentes de la matriz. Finalmente, en lo que respecta a los minerales presentes, la principal diferencia entre ambos grupos radica en la ya mencionada presencia de compuestos ferruginosos en la matriz, entre fragmentos del grupo B. Los minerales del grupo de la mica (biotita y muscovita) son frecuentes, especialmente entre los tiestos del grupo A, y el cuarzo se registra solo en dos ejemplares, uno perteneciente a cada grupo discriminado.

Características de las inclusiones. Las características asociadas a las inclusiones de los tiestos se encuentran sintetizadas en las Tablas 6 y 7 , compiladas a partir de las fichas normalizadas de cada corte delgado. Como los datos referidos a todos los atributos pueden consultarse allí, enfatizaremos aquí aquellos que nos proporcionan información para la problemática de interés particular.

También en este caso se observan porcentajes relativamente bajos de inclusiones (no superan el 30\%) en ambos conjuntos cerámicos, y se mantienen las tendencias - observadas para el caso de la matriz- de una ausencia de orientación de los componentes minerales entre los tiestos del grupo B y una mayor regularidad entre los del grupo A.

En lo que respecta a las características de forma (grado de redondez) y tamaño (grado de selección), a pesar de existir una cierta variabilidad interna en cada grupo, es posible notar algunas tendencias en cada componente. Los tiestos del grupo A presentan una mayor proporción de cristales subredondeados a redondeados (esfericidad alta) que los del grupo B, mientras que éste último se caracteriza por una mayor frecuencia de cristales angulosos a subangulosos. Se observa un contraste más claro en términos de las diferencias de tamaño, ya que todas las inclusiones del grupo B se caracterizan por un grado de selección pobre a muy pobre, mientras que en el grupo $\mathrm{A}$ son frecuentes los casos de selección buena a moderadamente buena. ${ }^{6}$

\footnotetext{
${ }^{6}$ Ambos atributos de los cristales se vinculan con el tipo de transporte experimentado, en términos del agente interviniente y la
}

Si nos referimos ahora a los componentes minerales mayoritarios, podemos decir que estos corresponden en ambos grupos cerámicos- a inclusiones félsicas, que incluyen principalmente al cuarzo y las plagioclasas $y$, en menor proporción, a los feldespatos potásicos. Son igualmente frecuentes los minerales del grupo de la mica -biotita y muscovita- por lo que la presencia de estos componentes abundantes no aporta mayores datos para establecer diferencias o semejanzas entre los dos grupos, o datos sobre su procedencia, aunque sí algunas de sus características. En este sentido, debemos destacar como un atributo prácticamente exclusivo de los tiestos del grupo A, la presencia de cristales de cuarzo, plagioclasas y feldespatos que presentan fracturas, maclas deformadas y/o extinción ondulosa muy marcada, que permite inferir una fuente origen que habría sufrido cierto grado de metamorfismo o deformación importante.

Otra diferencia que surge entre ambos conjuntos está relacionada con los minerales de menor frecuencia de registro. Se trata de la identificación de cristales de fluorita y turmalina asociados entre sí, presentes exclusivamente en las pastas de un conjunto de tiestos del grupo A.? También otros minerales de baja frecuencia han sido registrados exclusivamente entre tiestos del grupo A (ópalo, apatito y olivino), aunque solo en ejemplares aislados. Por otra parte, en concordancia con las diferencias señaladas -entre ambos grupos - en el color predominante de la matriz, la presencia de óxidos de hierro está reflejada en la presencia de hematita secundaria con un mayor predominio entre tiestos del grupo B.

Finalmente, los restantes minerales de baja frecuencia de aparición (circón, piroxeno y horblenda), al igual que la inclusión de tiesto molido en algunas pastas, han sido registrados entre tiestos de los dos grupos principales.

En relación a los litoclastos, se han identificado componentes de variado origen genético, aunque es posible señalar algunas tendencias. Entre los tiestos del grupo A,

distancia recorrida desde la fuente de origen (Fernando Sardi, com. pers. 2006; Schuster 2007).

7 Con respecto a su origen, la fluorita puede asociarse a rocas graníticas con cierto grado avanzado de diferenciación (evolución), y a pegmatitas y venas y filones de cuarzo hidrotermales de alta temperatura. También la turmalina es frecuente en pegmatitas y en metamórficas como ciertos esquistos y gneises. 


\begin{tabular}{|c|c|c|c|c|c|c|c|c|c|c|c|c|c|c|c|c|c|c|c|}
\hline \multicolumn{2}{|l|}{$\mathrm{N}^{\circ}$} & 62 & 47 & 48 & 74 & 90 & 91 & 130 & 194 & 255 & 264 & 3 & $4 / 1$ & $4 / 2$ & 14 & 24 & 27 & 43 & 46 \\
\hline \multirow{2}{*}{ DEN } & $\mathrm{D}$ & $x$ & & $\mathrm{x}$ & $\mathrm{X}$ & & $\mathrm{x}$ & $\mathrm{X}$ & $\mathrm{X}$ & & $\mathrm{X}$ & $\mathrm{X}$ & $\mathrm{x}$ & $\mathrm{X}$ & $\mathrm{X}$ & $\mathrm{X}$ & $\mathrm{x}$ & $\mathrm{x}$ & $\mathrm{X}$ \\
\hline & $\mathrm{P} / \mathrm{d}$ & & $\mathrm{X}$ & & & $\mathrm{X}$ & & & & $\mathrm{X}$ & & & & & & & & & \\
\hline \multirow{3}{*}{ ORIENT } & $\mathrm{S} / \mathrm{o}$ & $\mathrm{X}$ & $\mathrm{X}$ & & & & $x$ & $\mathrm{X}$ & & $\mathrm{X}$ & $\mathrm{x}$ & & & & & & & & $\mathrm{X}$ \\
\hline & $\mathrm{R} / \mathrm{P}$ & & & $\mathrm{x}$ & $\mathrm{X}$ & & & & $\mathrm{x}$ & & & $\mathrm{x}$ & & $\mathrm{x}$ & $\mathrm{X}$ & $\mathrm{X}$ & $\mathrm{x}$ & $\mathrm{X}$ & \\
\hline & $\mathrm{R} / \mathrm{t}$ & & & & & $\mathrm{x}$ & & & & & & & $\mathrm{x}$ & & & & & & \\
\hline \multirow{2}{*}{ T.I. } & $\mathrm{Mi}$ & $\mathrm{X}$ & $\mathrm{X}$ & $\mathrm{x}$ & $\mathrm{X}$ & $\mathrm{x}$ & $\mathrm{x}$ & $\mathrm{X}$ & $\mathrm{X}$ & $X$ & $\mathrm{X}$ & $\mathrm{x}$ & $\mathrm{x}$ & $\mathrm{X}$ & $\mathrm{X}$ & $\mathrm{X}$ & $\mathrm{x}$ & $\mathrm{x}$ & $\mathrm{X}$ \\
\hline & $\mathrm{Ti}$ & & $\mathrm{x}$ & & $\mathrm{x}$ & & & & & & & & $\mathrm{x}$ & & & & & $x$ & \\
\hline \multirow{5}{*}{$\begin{array}{c}\text { FORMA } \\
\text { (REDONDEZ) }\end{array}$} & An & $x$ & & & $\mathrm{X}$ & & & & & $X$ & & & $\mathrm{x}$ & & & & $\mathrm{x}$ & & \\
\hline & S/a & & $\mathrm{X}$ & $\mathrm{x}$ & $\mathrm{X}$ & $\mathrm{x}$ & $\mathrm{x}$ & $\mathrm{X}$ & & $\mathrm{X}$ & & $x$ & & $\mathrm{X}$ & $\mathrm{x}$ & $\mathrm{x}$ & $\mathrm{x}$ & $x$ & \\
\hline & $S / \mathrm{r}$ & $\mathrm{x}$ & $\mathrm{x}$ & $\mathrm{x}$ & & $\mathrm{x}$ & $\mathrm{x}$ & & $\mathrm{X}$ & $\mathrm{X}$ & $\mathrm{X}$ & & $\mathrm{x}$ & & $\mathrm{X}$ & & & $\mathrm{X}$ & $\mathrm{X}$ \\
\hline & $\operatorname{Re}$ & $\mathrm{X}$ & $\mathrm{X}$ & $\mathrm{x}$ & & & & & & & & & & & & & & & $\mathrm{X}$ \\
\hline & $\mathrm{M} / \mathrm{r}$ & & & & & & & & & & & & & & & & & & \\
\hline \multirow{5}{*}{$\begin{array}{c}\text { TAMAÑO } \\
\text { (SELECCIÓN) }\end{array}$} & $\mathrm{M} / \mathrm{b}$ & & & & & & & & & & & & & & & & $x$ & & \\
\hline & Bue & & & & $\mathrm{X}$ & & & & $\mathrm{X}$ & $\mathrm{X}$ & & & & $\mathrm{X}$ & & $\mathrm{X}$ & & $\mathrm{x}$ & \\
\hline & Mod & $\mathrm{X}$ & $\mathrm{X}$ & & & & & & & & $\mathrm{x}$ & $\mathrm{X}$ & & & & & & & \\
\hline & Pob & $\mathrm{x}$ & & $\mathrm{x}$ & & $\mathrm{x}$ & $\mathrm{x}$ & & & & & & & & & & & & $\mathrm{x}$ \\
\hline & $\mathrm{M} / \mathrm{p}$ & & & & & & & $\mathrm{X}$ & & & & & $\mathrm{x}$ & & & & & & \\
\hline \multirow{16}{*}{$\begin{array}{l}\text { INCLUSIONES } \\
\text { MINERALES }\end{array}$} & Q & $\mathrm{X}$ & $\mathrm{X}$ & $\chi$ & $\mathrm{X}$ & $\chi$ & $\mathrm{x}$ & $\mathrm{X}$ & $\mathrm{X}$ & $\chi$ & $\mathrm{X}$ & $\mathrm{X}$ & $\chi$ & $\chi$ & $\mathrm{X}$ & $\chi$ & $\mathrm{X}$ & $\chi$ & \\
\hline & Pl & $\mathrm{X}$ & $\mathrm{X}$ & $\mathrm{X}$ & $\chi$ & X & $\mathrm{X}$ & $\mathrm{X}$ & $\mathrm{X}$ & $\chi$ & $\mathrm{X}$ & $\mathrm{X}$ & $\mathrm{X}$ & $\mathrm{X}$ & X & $\chi$ & $\mathrm{X}$ & $\chi$ & X \\
\hline & $\mathrm{F} / \mathrm{K}$ & & $\mathrm{X}$ & $\mathrm{x}$ & $\chi$ & & & $\mathrm{X}$ & & $X$ & $\mathrm{X}$ & & & & & $\chi$ & $\mathrm{x}$ & & $\mathrm{X}$ \\
\hline & Mic & $\mathrm{X}$ & & & & & & & & & & & & & & $\chi$ & & & \\
\hline & Ort & $\mathrm{X}$ & & & & & & & & & & & & & & & & & \\
\hline & $\mathrm{Bi}$ & $x$ & $\mathrm{X}$ & $\mathrm{x}$ & $\mathrm{X}$ & $\mathrm{x}$ & $\mathrm{x}$ & $\mathrm{X}$ & $\mathrm{x}$ & & $\mathrm{X}$ & $x$ & $\mathrm{x}$ & & $\mathrm{x}$ & $\mathrm{X}$ & $\mathrm{x}$ & $x$ & $\mathrm{x}$ \\
\hline & $\mathrm{Mu}$ & & $\mathrm{x}$ & $\mathrm{x}$ & $\mathrm{X}$ & $\mathrm{x}$ & & $\mathrm{X}$ & $\mathrm{X}$ & $\mathrm{X}$ & $\mathrm{x}$ & $\mathrm{x}$ & $\mathrm{x}$ & $\mathrm{x}$ & $\mathrm{X}$ & & $\mathrm{X}$ & $\mathrm{x}$ & $\mathrm{X}$ \\
\hline & Flu & & $\mathrm{X}$ & $\mathrm{X}$ & & $\mathrm{X}$ & & & & & & & $\mathrm{x}$ & $\mathrm{X}$ & & & & & $\mathrm{X}$ \\
\hline & Tur & $x$ & $\mathrm{x}$ & $\mathrm{x}$ & & & & & & & & & $\mathrm{x}$ & $\mathrm{x}$ & & & & & \\
\hline & Cir & & $\mathrm{X}$ & $\mathrm{x}$ & & & & & & & & & & & & & & & \\
\hline & Pir & $\mathrm{X}$ & $\mathrm{X}$ & & & & & & & & & & $\mathrm{x}$ & & & & & & \\
\hline & Oli & $\mathrm{X}$ & & & & & & & & & & & & & & & & & \\
\hline & Hor & & & & & & $\mathrm{X}$ & & & & & & & & & & & & \\
\hline & Apa & & & & & & & & & & & & & & & & $\mathrm{x}$ & & \\
\hline & $\mathrm{Fe}$ & & & & & & & & & & & & $\mathrm{x}$ & & & & & & \\
\hline & Opa & & & & & & & & & & & & & & & & & & $\mathrm{x}$ \\
\hline \multirow{5}{*}{ LITICOS } & Met & & & & & $\mathrm{X}$ & & & & & & & & & & & & & \\
\hline & Cua & $\mathrm{X}$ & & & & & & & $\mathrm{X}$ & $\mathrm{X}$ & $\mathrm{X}$ & & & & & & $\mathrm{X}$ & & \\
\hline & Vol & & & & & & $\mathrm{X}$ & & & & & & & & & & & & \\
\hline & Gra & $\mathrm{X}$ & & & & & & $\mathrm{X}$ & & $\mathrm{X}$ & $\mathrm{X}$ & & & & $\mathrm{X}$ & $\mathrm{X}$ & & & $\mathrm{x}$ \\
\hline & $\mathrm{V} / \mathrm{v}$ & $\mathrm{X}$ & $\mathrm{x}$ & $\mathrm{x}$ & $\mathrm{X}$ & $\mathrm{X}$ & & & $\mathrm{X}$ & & & $\mathrm{x}$ & $\mathrm{x}$ & $\mathrm{x}$ & & $\mathrm{X}$ & $\mathrm{X}$ & & $\mathrm{X}$ \\
\hline \multicolumn{2}{|l|}{$\%$} & 14 & 18 & 32 & & 12 & & 25 & 15 & 15 & 15 & 20 & 30 & 30 & 17 & 25 & 7 & 10 & 25 \\
\hline
\end{tabular}

Tabla 6. Atributos de las inclusiones en tiestos del grupo A. Referencias: Densidad (DEN): D: denso; P/d.: poco denso. Orientación (ORIENT): S/o: sin orientación; R/p: regular parcial y R/t: regular total. Tipo de inclusiones (T.I.): Mi: minerales; Ti: tiestos. Forma: An: anguloso; S/a: subanguloso; S/r: subredondeado; Re: redondeado; M/r: muy redondeado. Selección por tamaño: M/b: muy buena; Bue: buena; Mod: moderadamente buena; Pob: pobre; M/p: muy pobre. Minerales: Q: cuarzo; Pl: plagioclasa; F/K: feldespato potásico; Mic: microclino; Ort: ortosa; Bi: biotita, Mu: muscovita; Flu: fluorita; Tur: turmalina; Cir: circón; Pir: piroxeno; Oli: olivino; Hor: horblenda; Apa: apatito; Fe: hierro; Opa: ópalo. Líticos: Met: metamórficos; Cua. Cuarcita; Vol: volcánicos; Gran: granitos y V/v: vidrio volcánico. 
es notable el componente volcánico, atestiguado en el registro frecuente de vitroclastos $(66,66 \%)$, y apoyado por la identificación de ciertos componentes minerales vinculados a rocas básicas (olivino, piroxeno) y la presencia de feldespatos calcosódicos zonados. Una proporción menor de este primer conjunto $(38,88 \%)$, pero mayoritaria entre ejemplares del grupo B $(55,55 \%)$, corresponde a componentes de origen ígneo plutónico, donde el mayor aporte provendría de rocas graníticas. La identificación de cristales de turmalina, de fluorita y la presencia de feldespatos potásicos de textura gráfica se asociarían con rocas graníticas con cierto grado avanzado de diferenciación, y a pegmatitas y venas hidrotermales de alta temperatura. Por último, la identificación de fragmentos líticos de cuarcita, señala la presencia de material procedente de una zona con aporte metamórfico, aunque estos componentes petrográficos son los más escasamente representados, en términos comparativos, en ambos conjuntos (33,33\%).

Analizamos a continuación los resultados de la caracterización petrográfica, principalmente en términos de aportar argumentos para la discusión de la hipótesis originalmente planteada, es decir, la procedencia foránea del conjunto de tiestos que han sido representados -en la muestra bajo análisis- por el grupo A.

\section{* Estableciendo semejanzas y diferencias ENTRE LOS DOS CONJUNTOS LOCALES}

Señalamos previamente algunas implicancias para la contrastación de la hipótesis básica, las que se apoyan principalmente tanto en la relativa homogeneidad y coherencia que debería observarse internamente en cada conjunto; como en la existencia de pautas de diferenciación o contraste entre ellos. Al respecto, podemos señalar que un conjunto de similitudes (intra-grupo) y diferencias (extra-grupo) observadas tanto en atributos de la matriz como de las inclusiones, permitirían sostener - preliminarmente- la segregación inicial entre los dos conjuntos cerámicos (A y B), basada hasta ahora exclusivamente en atributos macroscópicos, también desde un punto de vista composicional mineral. Estas corresponden, en el caso de la matriz, a las diferencias observadas en el color de las pastas, las que podrían vincularse con la presencia de minerales particulares (óxidos de hierro en el conjunto B) y a la regularidad relevada en la orienta- ción de las inclusiones, muy marcada en el conjunto A y ausente en el conjunto B. De todos modos, no podemos dejar de tomar estas diferencias con cierto recaudo, debido a que podrían vincularse con aspectos tecnológicos, tales como diferencias en las temperaturas y atmósferas de cocción de las piezas -que podrían alterar el color de la matriz- y en las técnicas empleadas para la manufactura (molido, amasado, modelado, etc.), que podrían influir en la mayor o menor orientación de los minerales (Schuster 2007). Estas diferencias podrían estar, a su vez, más vinculadas con distintos requerimientos morfo-tecnológicos de las piezas (morfología, dimensiones, funciones a cumplir, etc.), que con diferencias de origen, lo que estaría apoyado por las diferencias observadas en estos aspectos entre los fragmentos que conforman cada uno de los dos grupos (ver Tablas 2 y 3 ).

$\mathrm{Si}$ nos referimos a las inclusiones, es posible afirmar que -de acuerdo a los criterios de forma y tamaño- los tiestos del grupo A muestran una mayor incidencia de procesos de transporte que el grupo B, ya sea por la intervención de agentes de distinta naturaleza (fluvial, eólico, etc.) y/o por efecto de la distancia recorrida desde el lugar de origen o roca madre. En segundo término, hemos detectado atributos mineralógico-composicionales exclusivos de ejemplares del grupo A, tales como la presencia de fluorita asociada a turmalina y la identificación de cuarzos, plagioclasas y feldespatos potásicos con elevada deformación o metamorfismo; plasmada en frecuentes fracturas y una extinción ondulosa muy marcada. Aquí es interesante señalar que, si bien este criterio permite diferenciar ambos conjuntos entre sí, no es una característica que otorgue homogeneidad interna al grupo posiblemente foráneo, ya que no está presente en todos - ni siquiera en la mayoría - de los tiestos de este grupo. Sin embargo, nos parece relevante notar que permite vincular composicionalmente a un conjunto de fragmentos agrupados inicialmente bajo el mismo rótulo estilístico (Ciénaga), y que se presentan muy similares macroscópicamente. ${ }^{8}$ A la vez, permite discriminar este subconjunto de otros ejemplares, similares desde un punto de vista formal, que integran el conjunto mayor de tiestos A, en principio "vallistos".

8 Todos los ejemplares exhiben representaciones incisas y/o grabadas de motivos figurativos y zoomorfos, sobre la superficie pulida de pastas reductoras y compactas. 


\begin{tabular}{|c|c|c|c|c|c|c|c|c|c|c|}
\hline \multicolumn{2}{|l|}{$\mathrm{N}^{\circ}$} & 2 & 49 & 72 & 89 & 135 & 211 & 38 & 76 & 79 \\
\hline \multirow{2}{*}{ DEN } & D & $\mathrm{x}$ & $\mathrm{x}$ & $\mathrm{X}$ & $\mathrm{X}$ & $\mathrm{X}$ & $\mathrm{X}$ & & $\mathrm{X}$ & $\mathrm{X}$ \\
\hline & $\mathrm{P} / \mathrm{d}$ & & & & & & & $\mathrm{X}$ & & \\
\hline \multirow{3}{*}{ ORIENT } & S/o & $\mathrm{X}$ & $\mathrm{x}$ & $\mathrm{X}$ & $\mathrm{x}$ & & $\mathrm{X}$ & $\mathrm{X}$ & $\mathrm{X}$ & $\mathrm{X}$ \\
\hline & $\mathrm{R} / \mathrm{P}$ & & & & & & & & & \\
\hline & $\mathrm{R} / \mathrm{t}$ & & & & & & & & & \\
\hline \multirow{2}{*}{ T.I. } & $\mathrm{Mi}$ & $\mathrm{X}$ & $\mathrm{x}$ & $\mathrm{X}$ & $\mathrm{x}$ & $\mathrm{X}$ & $\mathrm{X}$ & $\mathrm{X}$ & $\mathrm{X}$ & $\mathrm{X}$ \\
\hline & $\mathrm{Ti}$ & $\mathrm{x}$ & $\mathrm{x}$ & & & & & $\mathrm{x}$ & & \\
\hline \multirow{5}{*}{$\begin{array}{c}\text { FORMA } \\
\text { (REDONDEZ) }\end{array}$} & $\mathrm{An}$ & $\mathrm{x}$ & & $\mathrm{X}$ & $\mathrm{X}$ & & $\mathrm{X}$ & & $\mathrm{X}$ & $\mathrm{x}$ \\
\hline & $\mathrm{S} / \mathrm{a}$ & $\mathrm{X}$ & $\mathrm{x}$ & $\mathrm{X}$ & $\mathrm{X}$ & $\mathrm{X}$ & $\mathrm{X}$ & $\mathrm{X}$ & $\mathrm{X}$ & $\mathrm{x}$ \\
\hline & $\mathrm{S} / \mathrm{r}$ & $\mathrm{X}$ & $\mathrm{x}$ & & & $\mathrm{X}$ & $\mathrm{x}$ & $\mathrm{x}$ & & $\mathrm{x}$ \\
\hline & $\mathrm{Re}$ & & & & & & & $\mathrm{X}$ & & \\
\hline & $\mathrm{M} / \mathrm{r}$ & & & & & & & & & \\
\hline \multirow{5}{*}{$\begin{array}{c}\text { TAMAÑO } \\
\text { (SELECCIÓN) }\end{array}$} & $\mathrm{M} / \mathrm{b}$ & & & & & & & & & \\
\hline & Bue & & & & & & & & & \\
\hline & Mod & & & & & & & & & \\
\hline & Pob & $\mathrm{x}$ & $\mathrm{x}$ & & & $\mathrm{X}$ & $\mathrm{X}$ & $\mathrm{x}$ & & \\
\hline & $\mathrm{M} / \mathrm{p}$ & & & $\mathrm{X}$ & $\mathrm{x}$ & & & & $\mathrm{X}$ & X \\
\hline \multirow{16}{*}{$\begin{array}{l}\text { INCLUSIONES } \\
\text { MINERALES }\end{array}$} & Q & $\mathrm{X}$ & $\mathrm{x}$ & $\mathrm{X}$ & $\mathrm{X}$ & $\mathrm{X}$ & $\mathrm{X}$ & $\mathrm{X}$ & $\mathrm{X}$ & $\mathrm{X}$ \\
\hline & $\mathrm{Pl}$ & $\mathrm{X}$ & & $\mathrm{X}$ & $\mathrm{X}$ & $\mathrm{X}$ & $\mathrm{X}$ & $\mathrm{X}$ & $\mathrm{X}$ & $\mathrm{X}$ \\
\hline & $\mathrm{F} / \mathrm{K}$ & & & & & $\mathrm{x}$ & & & & \\
\hline & Mic & $\mathrm{X}$ & & $\mathrm{X}$ & $\mathrm{X}$ & & $\mathrm{X}$ & & $\mathrm{X}$ & $\mathrm{X}$ \\
\hline & Ort & $\mathrm{X}$ & & & & & & $\mathrm{X}$ & & $\mathrm{X}$ \\
\hline & $\mathrm{Bi}$ & $\mathrm{X}$ & $\mathrm{X}$ & $\mathrm{X}$ & $\mathrm{X}$ & & $\mathrm{X}$ & $\mathrm{X}$ & $\mathrm{X}$ & $\mathrm{X}$ \\
\hline & $\mathrm{Mu}$ & $\mathrm{X}$ & & $\mathrm{X}$ & $\mathrm{X}$ & & $\mathrm{X}$ & & $\mathrm{X}$ & $\mathrm{X}$ \\
\hline & Flu & & & & & & & & & \\
\hline & Tur & & & & & & & & & \\
\hline & Cir & & & & & & & $\mathrm{X}$ & & $\mathrm{X}$ \\
\hline & Pir & & & & & & $\mathrm{X}$ & & & \\
\hline & Oli & & & & & & & & & \\
\hline & Hor & & & & & & $\mathrm{X}$ & & & \\
\hline & Аpa & & & & & & & & & \\
\hline & $\mathrm{Fe}$ & & & $\mathrm{X}$ & $\mathrm{X}$ & & & & $\mathrm{X}$ & \\
\hline & Opa & & & & & & & & & \\
\hline \multirow{5}{*}{ LITICOS } & Met & $\mathrm{x}$ & & & $\mathrm{x}$ & & & $\mathrm{X}$ & & \\
\hline & Cua & $\mathrm{X}$ & & & $\mathrm{X}$ & & $\mathrm{X}$ & & & \\
\hline & Vol & & $\mathrm{X}$ & & & & & & & \\
\hline & Gra & $\mathrm{x}$ & & $\mathrm{X}$ & & $\mathrm{X}$ & $\mathrm{X}$ & & $\mathrm{X}$ & \\
\hline & $\mathrm{V} / \mathrm{v}$ & $\mathrm{X}$ & & & & & & $\mathrm{X}$ & & X? \\
\hline \multicolumn{2}{|l|}{$\%$} & 26 & & & 25 & & 25 & 5 & & \\
\hline
\end{tabular}

Tabla 7. Atributos de las inclusiones en tiestos del grupo B. Referencias: Densidad (DEN): D: denso; P/d.: poco denso. Orientación (ORIENT): S/o: sin orientación; R/p: regular parcial y R/t: regular total. Tipo de inclusiones (T.I.): Mi: minerales; Ti: tiestos. Forma: An: anguloso; S/a: subanguloso; S/r: subredondeado; Re: redondeado; M/r: muy redondeado. Selección por tamaño: M/b: muy buena; Bue: buena; Mod: moderadamente buena; Pob: pobre; M/p: muy pobre. Minerales: Q: cuarzo; Pl: plagioclasa; F/K: feldespato potásico; Mic: microclino; Ort: ortosa; Bi: biotita, Mu: muscovita; Flu: fluorita; Tur: turmalina; Cir: circón; Pir: piroxeno; Oli: olivino; Hor: horblenda; Apa: apatito; Fe: hierro; Opa: ópalo. Líticos: Met: metamórficos; Cua. Cuarcita; Vol: volcánicos; Gran: granitos y V/v: vidrio volcánico. 
Respecto a los componentes líticos, en ambos conjuntos se observan mayormente elementos de origen volcánico y plutónico, predominado claramente los primeros entre los tiestos del conjunto A y los segundos en el B; siendo coincidentes en ambos grupos la presencia minoritaria de líticos de aporte metamórfico y la ausencia de material sedimentario.

En síntesis, si consideramos en conjunto todos los aspectos señalados, vemos que algunos de ellos se constituyen en líneas de evidencia que permitirían sostener la discriminación inicial entre los conjuntos inferidos como foráneo (A) y local (B), especialmente ciertas inclusiones minerales y sus características asociadas; a la vez que otros parámetros de diferenciación implican tener mayor recaudo en su interpretación. En este sentido, si bien los dos grupos muestran ciertas diferencias composicionales entre sí, no son totalmente homogéneos internamente desde el punto de vista mineralógico -especialmente el grupo A- por lo que consideramos que se necesita contar con mayores datos y análisis complementarios para avanzar en la resolución de la discusión planteada.

Con este fin, abordamos a continuación el análisis desde un plano composicional comparativo, para examinar qué conclusiones es posible extraer al comparar las muestras procedentes de ANS con la cerámica estilísticamente afín, pero recuperada en sitios de valles.

\section{* El plano comparativo. caracterización PETROGRÁFICA DE LAS MUESTRAS CERÁMicAs DE VALLE}

\section{La cerámica del valle de la Ciénaga y del valle de Abaucán}

De acuerdo con la hipótesis básica sostenida, los ejemplares correspondientes a nuestro grupo A deben ser similares, composicionalmente hablando, a los fragmentos cerámicos asignados a los mismos estilos pero que fueron recuperados en los sitios de valles.

Para emprender este análisis comparativo contamos, en primer término, con los datos composicionales brindados por Zagorodny y colaboradores (2008), quienes presentan la caracterización petrográfica de una muestra cerámica procedente del valle de la Ciénaga, a partir del análisis efectuado sobre un total de 60 cortes delgados. Concluyen los autores que los resultados del análisis petrográfico indican una correspondencia composicional con la sucesión sedimentaria descripta para la región en la que se encuentran emplazados los sitios arqueológicos, siendo altamente probable que las materias primas usadas para la confección de la cerámica sean de origen local (Zagorodny et al. 2008).

$\mathrm{Al}$ respecto, consideramos que los principales puntos en común entre las dos muestras señaladas al inicio de este acápite están dados por los siguientes aspectos. En primer lugar, la recurrencia en contenidos minerales de origen piroclástico (vulcanismo explosivo), al caracterizarse ambos conjuntos, con diferentes áreas de recuperación, por la presencia de abundantes vitroclastos (pumitas y trizas). También entre la muestra de La Ciénaga se incluyen componentes accesorios de origen volcánico, y líticos de origen plutónico y metamórfico, como elementos escasos o accidentales, así como la presencia de tiesto molido en muy pocos ejemplares. Otro elemento en común se relaciona con la identificación de cristales de cuarzo con extinción ondulante en una proporción no muy elevada de las muestras, aunque el porcentaje es significativamente menor entre los tiestos procedentes de valles. Se suma el hecho de que no se ha reportado, para la muestra recuperada en el valle de La Ciénaga, la presencia de ciertos minerales registrados entre tiestos del grupo A (fluorita, turmalina, ópalo, apatito y olivino), aunque sí la ocurrencia escasa de litoclastos de origen sedimentario (pelíticos), que en cambio están ausentes en la muestra procedente de ANS.

Por lo tanto, podemos señalar que ambos conjuntos cerámicos -formal y estilísticamente similares desde un punto de vista macroscópico-, pero con áreas geográficas de recuperación diferentes, muestran una composición petrográfica y mineralógica básica afín en cuanto a su origen aunque con algunas particularidades en sus pastas que los distinguen.

La segunda muestra comparativa procede de diferentes contextos -residenciales, funerarios, productivos y ceremoniales- asociados a sitios arqueológicos emplazados en el valle de Abaucán, los que están temporalmente vinculados a una extensa secuencia cronológica (Ratto et al. 
2005). Los resultados del análisis petrográfico indican un alto grado de similitud entre las muestras de valle y el conjunto puneño procedente de ANS, al estar ambos conjuntos dominados por minerales félsicos (cuarzo y feldespatos) y biotita, combinados en menor proporción con fragmentos de rocas, principalmente volcánicas, con menor presencia de graníticas y metamórficas. Asimismo, la presencia de inclusiones arcillosas y/o tiesto molido es baja. Aunque una diferencia estaría marcada por el hecho de que Ratto y colaboradores (2005) señalan que el vidrio volcánico y los minerales ferro-magnesianos - piroxenos, anfíboles y epidotos- se presentan en baja proporción entre los fragmentos de Abaucán. También en este caso los autores concluyen que estos resultados petrográficos son totalmente coherentes con el perfil geológico regional, existiendo una correlación positiva a nivel mineralógico con la composición de los fragmentos cerámicos analizados (Ratto et al. 2005).

Sobre la base de esta información, podemos concluir que un análisis comparativo basado exclusivamente en la caracterización petrográfica de las pastas, permite sugerir las probables áreas de aporte del material lítico y mineral pero no es posible precisar las fuentes de extracción de las materias primas. A ello sumamos que, entre los componentes minerales y líticos identificados en los cortes, no se detectaron inclusiones que puedan indicar de manera certera un área de procedencia determinada, ya que todos estos materiales forman parte de los componentes geológicos básicos de la región puneña, fundamentalmente en lo que respecta a la predominancia de inclusiones de origen volcánico extrusivo y plutónico. Un aspecto que no facilita el abordaje está dado por la similitud composicional existente entre los basamentos geológicos de ambas áreas. Nos referimos a la presencia de bloques de Basamento Cristalino, constituidos principalmente por rocas graníticas y, en menor escala, por migmatitas y rocas metamórficas inyectadas en distinto grado, asociados a la existencia de formaciones que incluyen componentes de origen volcánico y piroclástico, tanto en la Puna como en la zona valliserrana meridional (González Bonorino 1947, 1972; Hongn y Seggiaro 2001; Seggiaro 1999; Sosic 1972). En otros términos, y mientras no contemos con mayores datos, es posible plantear que las materias primas usadas para la confección de la cerámica "vallista" recuperada en ANS podrían haber sido obtenidas en la región de estudio. Es por ello que creemos necesario explorar a continuación los datos complementarios, obtenidos a través del empleo de otros procedimientos analíticos (AANI), que permitan superar la heterogeneidad inherente a la naturaleza del material cerámico y caracterizar a los distintos componentes de la matriz o arcilla.

\section{* Los anÁlisis de CARACTERIzación QUímica MUltielemental (AANi)}

\section{Conformación de la muestra de análisis}

Del conjunto original integrado por 27 tiestos que fueron caracterizados por corte delgado, 21 fragmentos fueron sometidos a AANI. ${ }^{9}$ No se duplicó la muestra completa, debido a las reducidas dimensiones de algunos ejemplares, que nos impidieron contar con fragmentos de tamaño y peso suficiente para su irradiación, luego de haberse realizado los cortes y montajes.

Para el análisis comparativo, se conformó una muestra cerámica en la que se seleccionaron 65 casos procedentes tanto de sitios de ANS como del valle de Abaucán. ${ }^{10}$ De este conjunto, un total de 31 tiestos integran la muestra local y proceden de los sitios PP9 I (17), PH2 (13), PP12 (1), y otros 10 fragmentos, procedentes de recolección superficial de estos mismos sitios de ANS, que habían sido analizados previamente por AANI por la Dra. Norma Ratto y equipo (2007).

Los 34 tiestos restantes, que son los que conforman la muestra comparativa, han sido recuperados en los sitios Ojo del Agua 1 (7); Palo Blanco NH3 (10) y NH6 (4); Tatón 1 (8) y La Troya LT-V5o (5). Se trata de asentamientos emplazados en diferentes sectores del Bolsón de Fiambalá, en el valle de Abaucán. Todos estos sitios han sido vinculados con una cronología asociada al período Formativo, en base a datos radiocarbónicos y/o contex-

\footnotetext{
9 Las muestras fueron preparadas y analizadas mediante Análisis por Activación Neutrónica Instrumental (AANI) en los laboratorios del Grupo Técnicas Analíticas Nucleares del Centro Atómico Ezeiza, Comisión Nacional de Energía Atómica (CNEA), Buenos Aires.

${ }^{10}$ Inicialmente, se seleccionaron 66 casos, aunque luego de efectuar la primera corrida del programa estadístico se detectó un outlier (núm. 38, sitio PH2), por lo que se retomó el análisis con un total de 65 casos de estudio (Figura 5).
} 


\begin{tabular}{|c|c|c|c|c|c|c|}
\hline \multirow{2}{*}{ Sitio Arqueológico } & \multicolumn{5}{|c|}{ Grupos Predictivos } & \multirow{2}{*}{ Total } \\
\hline & 1 & 2 & 3 & 4 & 5 & \\
\hline \multirow{2}{*}{$\mathrm{OA}_{1}$} & 4 & 3 & o & $\circ$ & ० & 7 \\
\hline & $57,14 \%$ & $42,86 \%$ & - & - & - & $10,77 \%$ \\
\hline \multirow{2}{*}{$\mathrm{PBN} \mathrm{H}_{3}$} & 2 & 6 & 1 & 1 & o & 10 \\
\hline & $20 \%$ & $60 \%$ & $10 \%$ & $10 \%$ & - & $15,38 \%$ \\
\hline \multirow{2}{*}{ PBN H6 } & 1 & 3 & ० & ० & o & 4 \\
\hline & $25 \%$ & $75 \%$ & - & - & - & $6,15 \%$ \\
\hline \multirow{2}{*}{$\mathrm{Tt} 1$} & 4 & 3 & o & 1 & o & 8 \\
\hline & $50 \%$ & $37,5 \%$ & - & $12,5 \%$ & - & $12,3 \%$ \\
\hline \multirow{2}{*}{ LT-V5O } & o & 5 & o & o & o & 5 \\
\hline & - & $100 \%$ & - & - & - & $7,7 \%$ \\
\hline \multirow{2}{*}{$\mathrm{PH}_{2}$} & 1 & o & 8 & 2 & 2 & 13 \\
\hline & $7,7 \%$ & - & $61,54 \%$ & $15,38 \%$ & $15,38 \%$ & $20 \%$ \\
\hline \multirow{2}{*}{ PP12 } & 1 & o & o & ० & o & 1 \\
\hline & $100 \%$ & - & - & - & - & $1,55 \%$ \\
\hline \multirow{2}{*}{$\mathrm{PP}_{9}$} & 3 & 3 & 1 & 5 & 5 & 17 \\
\hline & $17,65 \%$ & $17,65 \%$ & $5,88 \%$ & $29,41 \%$ & $29,41 \%$ & $26,15 \%$ \\
\hline \multirow{2}{*}{ Total } & 16 & 23 & 10 & 9 & 7 & \multirow{2}{*}{65} \\
\hline & $24,63 \%$ & $35,38 \%$ & $15,38 \%$ & $13,84 \%$ & $10,77 \%$ & \\
\hline
\end{tabular}

Tabla 8. Grupos predictivos con perfiles químicos multielementales similares, discriminados por sitio arqueológico de procedencia. Referencias: Sitios formativos del Valle de Abaucán: OA: Ojo del Agua; PBNH: Palo Blanco Núcleo Habitacional; Tt: Tatón; LT: La Troya. Sitios formativos de Antofagasta de la Sierra: PH: Piedra Horadada; PP: Punta de la Peña.

tuales, especialmente rasgos arquitectónicos y determinaciones estilísticas de los conjuntos cerámicos (Feely y Ratto 2008; Valero Garcés y Ratto 2005; Valero Garcés et al. 2007).

En síntesis, tanto la selección de los sitios arqueológicos, como del conjunto cerámico para el análisis comparativo, se basaron en los datos cronológicos disponibles para los rasgos y tecnofacturas aludidas. Hemos pretendido, de este modo, conformar una muestra cerámica que posea coherencia interna en lo que respecta a la sincronía de los eventos comparados.

\section{Resultados e interpretación del análisis de caracterización química}

Antes de profundizar en la problemática central de este trabajo, debemos señalar que el AD generó cinco grupos predictivos, cuyos elementos componentes se asocian en función de su grado de similitud en sus perfiles químicos multielementales. Una de las primeras observaciones que se desprenden de este análisis es que mientras los cuatro primeros grupos predictivos incluyen tiestos de ambas regiones, el quinto agrupamiento está conformado exclusivamente por fragmentos procedentes de sitios de ANS. Dentro de esta tendencia general, es posible también notar que la cerámica recuperada en los asentamientos de Abaucán es claramente mayoritaria en el grupo $1(68,75 \%)$ y absolutamente dominante en el segundo grupo $(86,95 \%)$. Por el contrario, una tendencia inversa se observa en los otros grupos combinados, donde los tiestos procedentes de ANS predominan claramente en el tercer (90\%) y cuarto grupo (más del 77\%) (Tablas 8 y 9 , Figuras 6 y 7 ).

Si retomamos el eje central de la discusión, los resultados del análisis químico multielemental deberían permitirnos comprobar si los dos grupos (A y B), inicialmente 


\begin{tabular}{|c|c|c|c|c|c|c|c|}
\hline \multirow{2}{*}{ Procedencia } & \multirow{2}{*}{$\begin{array}{c}\text { Estilo } \\
\text { Cerámico }\end{array}$} & \multicolumn{5}{|c|}{ Grupos Composicionales } & \multirow{2}{*}{ Total } \\
\hline & & 1 & 2 & 3 & 4 & 5 & \\
\hline \multirow{12}{*}{$\begin{array}{c}\text { Antofagasta } \\
\text { de la Sierra }\end{array}$} & \multirow{2}{*}{ Aguada } & O & o & 1 & O & O & 1 \\
\hline & & - & - & $100 \%$ & - & - & $3,22 \%$ \\
\hline & \multirow{2}{*}{ Ciénaga } & O & 2 & 6 & 3 & 2 & 13 \\
\hline & & - & $15,39 \%$ & $46,15 \%$ & $23,07 \%$ & $15,39 \%$ & $41,93 \%$ \\
\hline & \multirow{2}{*}{ Indeterminado } & o & o & o & o & 1 & 1 \\
\hline & & - & - & - & - & $100 \%$ & $3,22 \%$ \\
\hline & \multirow{2}{*}{ Saujil } & 5 & $\mathrm{O}$ & 2 & 4 & $\mathrm{O}$ & 11 \\
\hline & & $45,45 \%$ & - & $18,19 \%$ & $36,36 \%$ & - & $35,5 \%$ \\
\hline & \multirow{2}{*}{ Tosco } & $\circ$ & 1 & ○ & ○ & 4 & 5 \\
\hline & & - & $20 \%$ & - & - & $80 \%$ & $16,13 \%$ \\
\hline & \multirow{2}{*}{ Total } & 5 & 3 & 9 & 7 & 7 & \multirow{2}{*}{31} \\
\hline & & $16,13 \%$ & $9,67 \%$ & $29,04 \%$ & $22,58 \%$ & $22,58 \%$ & \\
\hline \multirow{12}{*}{ Valle de Abaucán } & \multirow{2}{*}{ Aguada } & 1 & 2 & 1 & $\circ$ & o & 4 \\
\hline & & $25 \%$ & $50 \%$ & $25 \%$ & - & - & $11,76 \%$ \\
\hline & \multirow{2}{*}{ Ciénaga } & 2 & 3 & ० & o & o & 5 \\
\hline & & $40 \%$ & $60 \%$ & - & - & - & $14,70 \%$ \\
\hline & \multirow{2}{*}{ Indeterminado } & $\circ$ & 1 & o & o & o & 1 \\
\hline & & - & $100 \%$ & - & - & - & $2,96 \%$ \\
\hline & \multirow{2}{*}{ Saujil } & 7 & 12 & ○ & 2 & 0 & 21 \\
\hline & & $33,33 \%$ & $57,14 \%$ & - & $9,53 \%$ & - & $61,76 \%$ \\
\hline & \multirow{2}{*}{ Tosco } & 1 & 2 & o & o & 0 & 3 \\
\hline & & $33,33 \%$ & $66,67 \%$ & - & - & - & $8,82 \%$ \\
\hline & \multirow{2}{*}{ Total } & 11 & 20 & 1 & 2 & 0 & \multirow{2}{*}{34} \\
\hline & & $32,35 \%$ & $58,82 \%$ & $2,96 \%$ & $5,87 \%$ & - & \\
\hline
\end{tabular}

Tabla 9. Grupos predictivos con perfiles químicos multielementales similares, discriminados por área de procedencia y estilo cerámico.

conformados en base a similitudes macroscópicas, se relacionan en su aspecto composicional químico y, a la vez, comparar estos resultados con los obtenidos a partir de la caracterización petrográfica. En este sentido, podemos comenzar diciendo que, en el caso de los tiestos interpretados como locales (grupo B), se observa una elevada correspondencia entre los distintos niveles de análisis, ya que, de la muestra total de tiestos de este grupo que fueron irradiados $(\mathrm{N}=7)$, solo uno no comparte un mismo grupo composicional. Los otros seis fragmentos se agrupan en un mismo grupo predictivo (grupo 5), que corresponde además al integrado exclusivamente por cerámica procedente de los sitios $\mathrm{PP} 9$ y $\mathrm{PH} 2$ de ANS. En este caso entonces, podemos afirmar que las semejanzas y diferencias observadas a nivel macroscópico y composicional petrográfico, presentan una correspondencia con el perfil químico multielemental de las piezas, confirmando la agrupación inicialmente planteada. ${ }^{11}$

11 Debemos decir que el único tiesto del grupo B que no comparte un mismo perfil químico con los restantes (núm. 49) muestra
En cambio, los ejemplares que conforman nuestro grupo en principio foráneo (A), muestran un comportamiento químico diferente, que nos permitiría cuestionar una supuesta homogeneidad composicional, planteada en base a un conjunto de atributos técnicos y estilísticos similares. Al respecto, recordemos que esta observación ya había sido anticipada en base a algunos de los resultados desprendidos del análisis petrográfico. Esto se refleja en el hecho que, del conjunto total irradiado $(\mathrm{N}=14)$, solo una parte -aunque corresponde a la mayor proporción $(42,85 \%)$ - integra un mismo conglomerado (grupo 3)

diferencias a nivel macroscópico, por presentar numerosas inclusiones líticas de color oscuro, que contrastan sobre una pasta de color rosado uniforme; no habiendo registrado otros fragmentos similares a éste en términos de su apariencia en los sitios trabajados. Aunque no es el único de su grupo, tampoco muestra evidencias de exposición al fuego, como la mayoría de los ejemplares que conforman el conjunto B. Desde el punto de vista petrográfico, es el único tiesto de la muestra total en el que no se han identificado plagioclasas que, junto con el cuarzo, constituyen los dos minerales dominantes. 


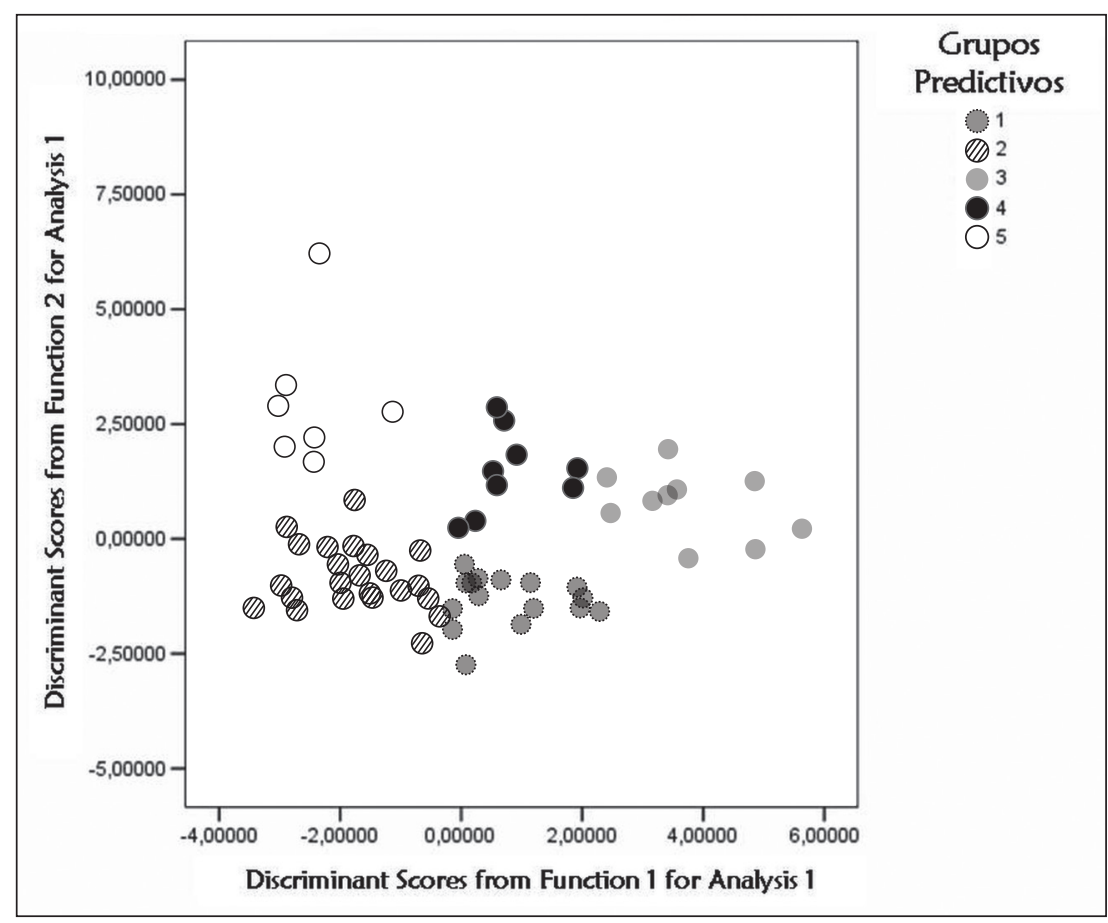

Figura 6. Representación de las funciones discriminantes (AD). Grupos predictivos con perfiles químicos multielementales similares.

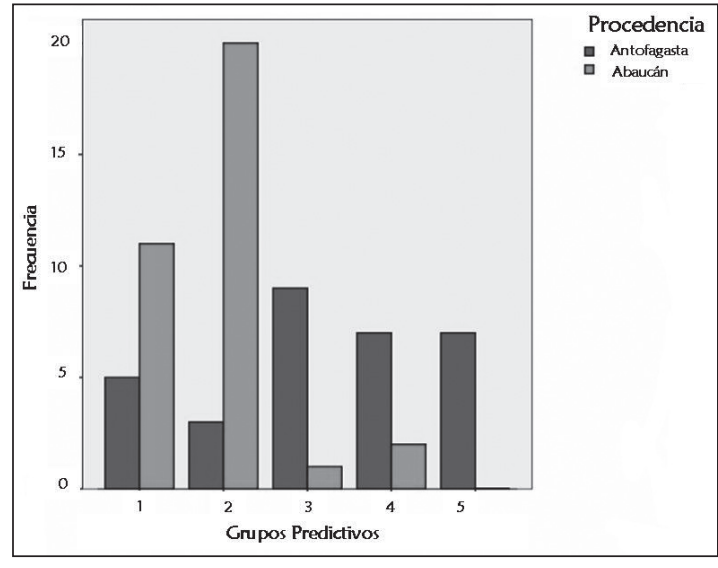

Figura 7. Frecuencia de los grupos predictivos con perfiles químicos multielementales similares, discriminados por región de procedencia.

que, como anticipamos, está mayoritariamente conformado por ejemplares procedentes de sitios locales, correspondientes a diferentes estilos interpretados como foráneos (Ciénaga, Saujil y Aguada). Los casos restantes que conforman nuestro grupo A o foráneo se reparten, de manera más o menos proporcional, entre los otros cuatro grupos predictivos generados por el $\mathrm{AD}$, es decir, vinculándose tanto con aquellos donde predominan los ejemplares procedentes de sitios del Valle de Abaucán (grupos 1 y 2), como con los formados mayoritaria (grupo 4) y exclusivamente (grupo 5) por tiestos recuperados en los sitios puneños donde, en el último caso, dominan ampliamente los ítems definidos como "toscos", e interpretados como de manufactura local. En conclusión, para el grupo inicialmente definido como foráneo (A), podemos afirmar que algunos ejemplares que mostraban ciertas diferencias desde un punto de vista petrográfico, comparten un mismo perfil químico, a la vez que se observan situaciones en las que cortes petrográficos que mostraban vínculos en base a sus inclusiones minerales, guardan perfiles composicionales químicos diferentes.

Podemos entonces concluir que los resultados obtenidos a partir del análisis químico multielemental permiten sostener, con elementos adicionales, algunas de las interpretaciones desprendidas del análisis macroscópico y petrográfico y, en otros casos, muestran diferencias en el perfil químico de tiestos afines en su apariencia y en su 
composición mineralógica; lo que pone de manifiesto un panorama de mayor complejidad que el hasta ahora bosquejado en las interpretaciones arqueológicas dominantes en relación a estos estilos cerámicos unificados bajo el rótulo de "valliserranos". A la vez, nos permiten contar con mayores elementos para inferir que los principales caracteres de homogeneidad que remarcamos anteriormente entre los ejemplares del grupo A (el color pardo de la matriz y la frecuente regularidad en la orientación de las inclusiones), estarían relacionados con aspectos tecnológicos productivos de las piezas, que exhiben tipos de pastas, atmósferas de cocción, tratamientos de superficie y atributos morfológicos y dimensionales similares (Tabla 2). Otros atributos mineralógicos compartidos entre algunos ejemplares del grupo A, como los de forma y selección por tamaño de las inclusiones, o la deformación en las maclas y fracturas frecuentes observadas en algunos minerales podrían, tentativamente, explicarse también como producto del procesamiento de las arcillas y/o las temperaturas de cocción alcanzadas (Schuster 2007), aunque otros, como la presencia asociada de minerales singulares (especialmente fluorita, turmalina) en ejemplares que en algunos casos se agrupan juntos, pero que en otros se separan en diferentes conglomerados químicos, son difíciles de explicar, y nos plantean la dificultad de trazar vínculos composicionales, entre las piezas cerámicas, solamente a partir de un análisis a nivel petrográfico.

\section{* A modo de conclusión: gentes y productos EN MOVIMIENTO CONSTANTE}

Si retomamos la hipótesis inicialmente planteada -que propone un origen foráneo para las piezas cerámicas recuperadas en sitios de la Puna de ANS, que fueron asociadas a "estilos valliserranos" (Ciénaga, Saujil, Aguada)-y la serie de implicancias enumeradas para su contrastación, podemos decir que los resultados alcanzados por los distintos niveles de análisis nos permiten aportar elementos para su discusión así como para una -creemosconveniente reconsideración. Al respecto, podemos decir que las expectativas de una homogeneidad (interna) y diferenciación (externa), para los dos grupos definidos para el análisis (A y B), solo se cumplen parcialmente. ${ }^{12}$

\footnotetext{
${ }^{12}$ Un punto a destacar es el hecho de que - a nivel de AANI- solo hemos podido comparar la muestra de Antofagasta de la Sierra
}

Esto es así para la casi totalidad (y la excepción ha sido explicada previamente) de los tiestos asimilados al grupo B o local, que conforman un conjunto afín composicionalmente, desde un punto de vista petrográfico y químico, además de nuclearse en un agrupamiento integrado exclusivamente por piezas de procedencia local. Esto nos permitiría confirmar para el conjunto de tiestos "toscos" nuestra división inicial, basada en criterios de similitud macroscópica y sostener, al menos con estas evidencias, su interpretación como piezas de elaboración local. En cuanto al conjunto cerámico $\mathrm{A}$, en principio foráneo, la primera conclusión que podemos extraer es que no se observa una homogeneidad composicional compartida por todos los ejemplares, expectativa que basamos en su correspondencia con estilos que han sido definidos para el área valliserrana del Noroeste Argentino. En otras palabras, solo algunos de estos fragmentos han podido ser vinculados macro y microscópicamente, ya que forman parte de las distintas agrupaciones que han sido discriminadas estadísticamente (AD), de acuerdo con sus atributos químicos composicionales. Entonces, si nos basamos en la información desprendida de los AANI, respecto de los perfiles químicos composicionales que posee la matriz o arcilla que conforma los tiestos, es posible afirmar que algunos ejemplares, que guardan una importante similitud formal desde el punto de vista tecnológico y estilístico (grupo A), habrían sido manufacturados con materias primas diferentes. A la vez, en algunos otros casos, ciertos tiestos que fueron discriminados a causa de sus notables discrepancias morfoestilísticas (grupos A y B), muestran una afinidad composicional, destacando aquí el caso de los dos tiestos Ciénaga que forman parte del mismo grupo predictivo (grupo 5) que la muestra de tiestos "toscos" o locales puneños. Es decir, podemos bosquejar un panorama donde, en algunos casos, una intención de "imitar" la apariencia final de ciertas vasijas, o bien una misma "manera de hacer" (Stark 1999), o una tradición tecnológica común, o un conocimiento artesanal alfarero compartido, involucra diferentes lugares de manufactura o fuentes de materia prima. ${ }^{13}$ Esto se da par-

con los tiestos procedentes del valle de Abaucán, y no con los recuperados en el valle de La Ciénaga, lo que en cierto modo puede representar una limitante en el análisis comparativo, que pensamos necesaria de salvar en estudios futuros.

${ }^{13} \mathrm{Al}$ respecto, son interesantes algunas de las conclusiones a las que arriba V. Schuster (2007), al analizar petrográficamente cortes delgados de cerámica procedente de áreas residenciales del sitio 
ticularmente para el caso de los estilos cuyo procedencia valliserrana se ha puesto aquí en consideración, los que, en algunos casos forman un grupo diferenciado composicionalmente de la muestra total de tiestos puneños y de los procedentes del valle de Abaucán (grupo 3), en otros se integran a los grupos predominantemente de valle (grupos 1 y 2), y asimismo se vinculan con la muestra exclusivamente puneña (grupo 5).

En términos de interpretar estos resultados $-y$ siempre con los recaudos que implica el haber trabajado con una muestra reducida- podemos decir que es posible comenzar a delinear un panorama de mayor diversidad que el que se percibe bajo la uniformidad que otorga el rótulo unificador de "estilos valliserranos" o "cerámica de valles", usado para nombrar a estas tecnofacturas recuperadas con frecuencia en sitios de la Puna. Creemos que esta reflexión es relevante en términos de que, como detallamos al inicio de este trabajo, diversas interpretaciones sobre la dinámica de las poblaciones que habitaron ambas áreas se sustentaron en estas similitudes estilísticas macroscópicas. Al respecto, si retomamos la reflexión de Aschero (2007: 100) sobre el posible origen de estas cerámicas - más allá del sesgo de género implícito en la presunción de que la producción alfarera fue una actividad exclusivamente femenina, y su consecuente implicancia de que fue la población puneña masculina la que estableció vínculos matrimoniales con las esponsales llegadas desde paisajes distantes- nos parece una propuesta sumamente interesante explorar el hecho de que ciertas piezas cerámicas exóticas, así como las producidas localmente, pero asimilables a estilos foráneos, puedan vincularse con la procedencia no local de los/las esponsales que residían en ANS. ${ }^{14}$ A su vez, concretar

Tebenquiche Chico (Antofalla). La autora (ibidem: 75) concluye que piezas similares macroscópicamente o bien incorporadas dentro de una misma categoría estilística - no local en todos los casos- exhiben composiciones mineralógicas diferentes en sus pastas. Esto le permite proponer una diversidad de posibles lugares de manufactura para piezas formalmente similares y una eventual "imitación" de estos atributos visuales en diversos lugares.

${ }^{14}$ Sin pretender hacer una lectura lineal hacia el pasado prehispánico, nos parece interesante mencionar que, a partir de los Registros Parroquiales locales que datan del siglo XIX, es posible inferir frecuentes vínculos matrimoniales entre pobladores antofagasteños y residentes de los valles próximos, como por ejemplo Fiambalá, Belén, Saujil, Molinos, Laguna Blanca, entre otros (García y Rolandi 2003). estos matrimonios entre pobladores de residencia lejana puede haber representado uno de los múltiples intereses de los viajes de interacción a larga distancia, tal como ha sido sugerido también por Haber (2007). Desde esta perspectiva, podemos proponer una mirada más amplia que la más frecuentemente esbozada hasta ahora para explicar un registro cerámico caracterizado por una alta frecuencia de tipos tecnológicos asociados a los "estilos valliserranos". Y así, más que pensar en un proceso marcado por influencias, desplazamientos $y / 0$ avances de grupos, desde el área valliserrana hacia la Puna, que se darían a partir de un determinado momento del devenir histórico de estas poblaciones (cercanos al comienzo de la era), vislumbramos en cambio una historia continua y mucho más antigua de interacciones entre la gente que habitaba estos distintos paisajes. Consideramos que este tipo de vínculos ancestrales, que reforzaban las relaciones a distancia, habrían permitido sostener una dinámica social que articulaba formas de acceso a los recursos sostenidas a través de nexos familiares (Aschero 2007; López Campeny et al. 2011).

Si bien en el estado de conocimiento actual creemos que la situación expuesta es muy compleja -y a la vez fragmentaria- como para permitirnos realizar afirmaciones concluyentes respecto de la procedencia de los conjuntos cerámicos, creemos que esta nueva información composicional abre un panorama que vislumbra una mayor diversidad de situaciones y prácticas en el pasado, y suscita nuevos interrogantes pero, sobre todo, ofrece una base material novedosa para el área de estudio, a partir de la cual es posible comenzar a discutir la tan asumida $-y$ poco cuestionada- procedencia exclusivamente vallista de estos componentes cerámicos recuperados en diversos contextos de la Puna Meridional argentina.

Agradecimientos A Carlos Aschero, mi director de Tesis y Beca Doctoral, así como de los distintos Proyectos -financiados por CONICET, Agencia y CIUNT- en el marco de los cuales se realizó la presente investigación; por la confianza y la libertad de trabajo que siempre sentí de su parte. A Bárbara Balesta, mi codirectora de Tesis, por su permanente apoyo y consejo, y por involucrarse en todo sentido en el análisis composicional cerámico, pese a la distancia física. A Nora Zagorodny, por proporcionar la ficha para las descripciones del material cerámico y por su interés y colaboración. A Fernando Sardi 
y Miguel Báez, por la suma responsabilidad y rapidez con que concretaron los análisis de los cortes delgados. A Norma Ratto, por su paciente asesoramiento para la clasificación estilística de la muestra cerámica, su invalorable asistencia durante la etapa de procesamiento y validación estadística de los datos y el posterior análisis multivariado, y por responder a las frecuentes dudas que toda esta etapa del análisis originó. También por su lectura crítica y recomendaciones para mejorar el texto. A
Raúl Zelaya por su paciente dedicación a los dibujos de los fragmentos cerámicos. A todos los que participaron de las excavaciones en PP9 y $\mathrm{PH} 2$, por su entusiasmo y dedicación durante las jornadas de trabajo. Finalmente, y no por ello menos importante, a dos revisores anónimos, por los acertados comentarios que permitieron mejorar una versión anterior del trabajo, aunque soy la única responsable de lo aquí expresado. A Tiago y Andrés, mis amores, por ser y estar.

\section{* Referencias citadas}

ASCHERO, C. A., 2006. De cazadores y pastores. El arte rupestre de la modalidad Río Punilla en Antofagasta de la Sierra y la cuestión de la complejidad en la Puna Meridional Argentina. En Tramas en la piedra: Producción y usos del arte rupestre, D. Fiore y M. Podestá (Eds.), pp. 103-140. World Archaeological Congress, Sociedad Argentina de Antropología y Asociación Amigos del INAPL.

2007. Comentario. Mesa 1: Interacciones surandinas. Aspectos económicos, políticos e ideológicos. En Sociedades precolombinas surandinas: Temporalidad, interacción y dinámica cultural del NOA en el ámbito de los Andes Centro-Sur, V. Williams, B. Ventura, A. Callegari y H. Yacobaccio (Eds.), pp. 99-108, Buenos Aires.

ASCHERO, C. A., R. ZURITA, M. G. COLANERI y A. TOSELLI, 2002. El bebé de la Peña.Actas XIII Congreso Nacional de Arqueología Argentina, tomo 2:329-336. Universidad Nacional de Córdoba.

BABOT, M. del P., 2004. Tecnología y utilización de artefactos de molienda en el Noroeste Prehispánico. Tesis de doctorado, Universidad Nacional de Tucumán, Tucumán.

BABOT, M. del P., C. A. ASCHERO, S. HOCSMAN, C. HAROS, L. GONZÁLEZ BARONI y S. V. URQUIZA, 2006. Ocupaciones agropastoriles en los Sectores Intermedios de Antofagasta de la Sierra (Catamarca): Un análisis desde Punta de la Peña 9.I. Comechingonia Revista de Arqueología 9: 57-75.

BABOT, M. del P., L. GONZÁLEZ BARONI, S. V. URQUIZA, M. G. AGUIRRE, M. G. COLANERI, S. HOCSMAN y C. HAROS, 2009. Dinámicas de formación y transformación de un entierro en el desierto puneño (Antofagasta de la Sierra, Puna Meridional Argentina). Intersecciones en Antropología 10: 183-201.

COHEN, M. L., 2005. Entre guano y arena... Ocupaciones recurrentes: Un caso de estudio en el sitio Punta de la Peña 9 III, Antofagasta de la Sierra, Catamarca. Trabajo Final de Carrera de Arqueología, Universidad Nacional de Tucumán, Tucumán.
CREMONTE, M. B., 1996. De las pastas a los olleros del pasado. En Revista XXVAniversario Museo Arqueológico Dr. Eduardo Casanova, pp. 47-51. Tilcara, Jujuy.

ESCOLA, P. S., 2007. Obsidianas en contexto: tráfico de bienes, lazos sociales y algo más. En Sociedades precolombinas surandinas: Temporalidad, interacción y dinámica cultural del NOA en el ámbito de los Andes Centro-Sur, V. Williams, B. Ventura, A. Callegari y H. Yacobaccio (Eds.), pp: 73-87. Kan sasana Printer, University of Michigan, Michigan.

ESCOLA, P. S., y S. HOCSMAN, 2008. Circulación macrorregional de un diseño artefactual en contextos agropastoriles: el caso de los cuchillos/raederas de módulo grandísimo. En Arqueología de las Tierras Altas de Argentina. Evolución y cambio cultural, H. Muscio y G. López (Eds.), BAR International Series.

FEELY, A., y N. RATTO, 2008. Variaciones de los conjuntos cerámicos de unidades domésticas: aldeas y puestos formativos del bolsón de Fiambalá (ca. 1500-1300 AP). En Entrelazando ciencias: sociedad y ambiente antes de la conquista española. EUDEBA, Buenos Aires.

GARCÍA, S., y D. ROLANDI, 2003. Antofagasta de la Sierra, Provincia de Catamarca. Su historia en los documentos y la tradición oral. En Puna de Atacama. Sociedad, economía y frontera, A. Benedetti (Comp.), pp. 137-197. Alción Editora, Córdoba.

GONZÁLEZ, A. R., 1977. Arte Precolombino de la Argentina. Filmediciones Valero, Buenos Aires.

GONZÁLEZ BONORINO, F., 1947. Descripción Geológica de la Hoja Geológica 12d, Capillitas, Provincia de Catamarca, República Argentina. Escala 1: 200.000. Ministerio de Industria y Minería, Subsecretaría de Minería, Dirección Nacional de Geología y Minería. 
1972. Descripción Geológica de la Hoja Geológica 13c, Fiambalá, Provincia de Catamarca, República Argentina. Escala 1:200.000. Ministerio de Industria y Minería, Subsecretaría de Minería, Dirección Nacional de Geología y Minería.

GRANIZO, M. G., 2001. La cerámica en Tebenquiche Chico. Una propuesta de interpretación categorial. Tesis de licenciatura, Escuela de Arqueología, Universidad Nacional de Catamarca.

HABER, A., 2001. El oasis en la articulación del espacio circumpuneño. Actas del XIII Congreso Nacional de Arqueología, tomo 1: 251267, Universidad Nacional de Córdoba, Córdoba.

2006. Una arqueología de los oasis puneños. Domesticidad, interacción e identidad en Antofalla. Primer y segundo milenios DC. Universitaslibros, Córdoba, en coedición con la Universidad de Cauca, Colombia.

2007. Comentarios marginales. En Sociedades precolombinas surandinas: Temporalidad, interacción y dinámica cultural del NOA en el ámbito de los Andes Centro-Sur, V. Williams, B. Ventura, A. Callegari y H. Yacobaccio (Eds.), pp. 59- 72, Kan sasana Printer, University of Michigan, Michigan.

HOCSMAN, S., 2006. Producción lítica, variabilidad y cambio en Antofagasta de la Sierra (ca. 5500-1500 AP). Tesis de doctorado, Universidad Nacional de la Plata.

HOCSMAN, S., J., G. MARTÍNEZ, M. F. RODRÍGUEZ y C. A. ASCHERO, 2004. Obtención de recursos distantes en la porción meridional de los Andes centro-sur: Una visión desde la Puna Argentina. En Before Farming, en prensa.

HONGN, F., y R. SEGGIARO, 2001. Hoja Geológica 2566-III, Cachi, Provincias de Salta y Catamarca. Programa Nacional de Cartas Geológicas 1:250.000, Subsecretaría de Minería de la Nación, Servicio Geológico Minero Argentino, Instituto de Geología y Recursos Minerales.

KRAPOVICKAS, P., 1955. El yacimiento Tebenquiche (Puna de Atacama). Publicaciones del Instituto de Arqueología III, Universidad de Buenos Aires, Buenos Aires.

LÓPEZ CAMPENY, S. M. L., 20oo. Tecnología, iconografía y ritual funerario. Tres dimensiones de análisis de los textiles formativos del Sitio Punta de la Peña 9 (Antofagasta de la Sierra, Argentina). Estudios Atacameños 20: 29-65.

2001. Actividades Domésticas y Organización del Espacio Intrasitio. El Sitio Punta de la Peña 9 (Antofagasta de la Sierra, Prov. de Catamarca). Trabajo Final de Carrera de Arqueología, Universidad Nacional de Tucumán, Tucumán.
2009. Asentamiento, Redes Sociales, Memoria e Identidad. Primer milenio de la era. Antofagasta de la Sierra, Catamarca. Tesis de doctorado, Universidad de La Plata, La Plata.

2010. De un hogar en la Puna... Relatos de idas y vueltas. En El hábitat prehispánico. Arqueología de la arquitectura y de la construcción del espacio organizado, M. E. Albeck, C. Scattolin y M. A. Korstanje (Eds.), pp. 215-242. Editorial de la Universidad Nacional de Jujuy, Jujuy.

LÓPEZ CAMPENY, S. M. L., E. DEL BEL, S. RODRÍGUEZ CURLETTO y A. S. ROMANO, 2005. Evidencias de ritualidad en contextos agropastoriles: el sitio Piedra Horadada 2 ( $\mathrm{PH} 2$ ), Puna Meridional Argentina. Serie Monográfica y Didáctica 45: 19 .

LÓPEZ CAMPENY, S. M. L., y P. S. ESCOLA, 2007. Un verde horizonte en el desierto: producción de cuentas minerales en ámbitos domésticos de sitios agropastoriles, Antofagasta de la Sierra (Puna Meridional Argentina). En Producción y circulación prehispánicas de bienes en el Sur Andino, A. Nielsen, C. Rivolta, V. Seldes, M. Vázquez y P. Mercolli (Comps.), tomo 2, pp. 225-258. Editorial Brujas, Córdoba.

LÓPEZ CAMPENY, S. M. L., A. ROMANO, M. F. RODRÍGUEZ, M. H. CORBALÁN y Á. R. MARTEL, 2011. De lazos familiares a redes sociales: nuevos aportes para la discusión de la interacción entre poblaciones de la Puna y las tierras bajas orientales. Ponencia presentada en el III Taller Internacional de Arqueología del NOA y Andes Centro Sur (TANOA III) "Arqueología y etnohistoria de la vertiente oriental de los Andes de Argentina, Bolivia y Perú", Museo López Pasquini, Alto La Viña, San Salvador de Jujuy.

OLIVERA, D. E., 1992. Tecnología y estrategias de adaptación en el Formativo (Agro-alfarero Temprano) de la Puna Meridional Argentina. Un caso de estudio: Antofagasta de la Sierra (Pcia. de Catamarca, R.A.). Tesis de doctorado, Universidad Nacional de La Plata, La Plata.

OLIVERA, D. E., y M. M. PODESTÁ, 1993. Los recursos del arte: arte rupestre y sistemas de asentamiento - subsistencia formativos en la Puna Meridional Argentina. Arqueología 3: 93 -126.

OLIVERA, D. E., y S. VIGLIANI, 2000-02. Proceso cultural, uso del espacio y producción agrícola en la Puna meridional argentina. Cuadernos del INAPL 19: 459-481.

PODESTÁ, M. M., y L. M. MANZI, 1995. Arte rupestre e interacción interregional en la Puna argentina. Cuadernos del INAPL 16: 367-399. 
RATTO, N., 2007. Paisajes arqueológicos en el tiempo: la interrelación de ciencias sociales, físico-químicas y paleoambientales (Dpto. Tinogasta, Catamarca, Argentina). En Producción y circulación prehispánicas de bienes en el Sur Andino, A. Nielsen, C. Rivolta, V. Seldes, M. Vázquez y P. Mercolli (Comps.), tomo 2, pp. 35-54. Editorial Brujas, Córdoba.

RATTO, N., M. ORGAZ, G. DE LA FUENTE y R. PLÁ, 2002. Ocupación de pisos de altura y contexto de producción cerámica durante el formativo: el caso de la región puneña de Chaschuil y su relación con el Bolsón de Fiambalá (Dpto. Tinogasta, Catamarca, Argentina). Estudios Atacameños 24: 51-69.

RATTO, N., S. QUENARDELLE y A. FEELY, 2005. Caracterización petrográfica de pastas cerámicas arqueológicas del Bolsón de Fiambalá (Dpto. Tinogasta, Catamarca). Actas del XVI Congreso Geológico Argentino, tomo IV: 151: 156.

RATTO, N., A. FEELY y R. PLÁ, 2007. La producción alfarera en el Bolsón de Fiambalá (Dpto. Tinogasta, Catamarca) y su alcance extra-regional. En Cerámicas arqueológicas: Perspectivas arqueométricas para su análisis e interpretación, B. Cremonte y N. Ratto (Eds.), pp. 123-146 y CD con figuras. Editorial de la Universidad Nacional de Jujuy, Jujuy.

RODRÍGUEZ, M. F., y J. MARTÍNEZ, 2001. Especies vegetales alóctonas como recursos arqueológicos en el ámbito puneño. $P u-$ blicación especial de la Asociación Paleontológica Argentina, 8: 139-145.

SANHUEZA, L., F. FALABELLA, E. FONSECA y O. ANDONIE, 2004. Aplicación de análisis de pastas macroscópicos, petrográficos y de composición de elementos químicos al problema de la procedencia de cerámica en el período Alfarero Temprano de Chile central y Cuyo, Argentina. Estudios Atacameños 28: 121-132.

SCHUTER, V., 2007. Petrografía de la cerámica de Tebenquiche Chico (Puna de Atacama). La Zaranda de Ideas 3: 57-78.

SEGGIARO, R., 1999. Hoja Geológica 2769-II, Paso de San Francisco, Provincia de Catamarca. Programa Nacional de Cartas Geológicas 1:250.000. Boletín 294, Subsecretaría de Minería de la Nación, Servicio Geológico Minero Argentino, Instituto de Geología y Recursos Minerales.

SEMPÉ, M. C., 1977. Caracterización de la cultura Saujil. Obra del Centenario del Museo de La Plata II: 211-235.
1993 Principios normativos del estilo de decoración en la cerámica Ciénaga. Publicaciones núm. 20 del Instituto de Investigaciones Arqueológicas y Museo. Facultad de Humanidades y Artes, Universidad de San Juan, San Juan.

SOSIC, M. V. J., 1972. Descripción Geológica de la Hoja Geológica 14d Tinogasta, Provincia de Catamarca, República Argentina. Escala 1:200.000. Ministerio de Industria y Minería, Subsecretaría de Minería, Dirección Nacional de Geología y Minería.

STARK, M., 1999. Social Dimensions of Technical Choice in Kalinga Ceramic Traditions. En Material Meaning. Critical Approaches to the Interpretation of Material Culture, E. Chilton (Ed.), pp. 61-84. The University of Utah Press, Salt Lake City.

TCHILINGUIRIÁN, P., y D. E. OLIVERA, 2000. De aguas y tierras: aportes para la reactivación de campos agrícolas arqueológicos en la Puna Argentina. Relaciones de la Sociedad Argentina de Antropología, tomo XXV: 99-118.

VALERO GARCÉS, B., y N. RATTO, 2005. Registros lacustres holocénicos en la puna de Chaschuil y el Bolsón de Fiambala (Dpto. Tinogasta, Catamarca): Resultados preliminares. Actas del XVI Congreso Geológico Argentino, tomo IV: 163-170.

VALERO GARCÉS, B., N. RATTO, A. MORENO, A. NAVAS y A. DELGADO HUERTAS, 2007. Los lagos del Altiplano de Atacama y el Noroeste Argentino como sensores de cambios hidrológicos durante el Holoceno. En Escenarios de Cambio Ambiental: Registros del Cuaternario en América Latina, M. Caballero (Ed.). Unión Mexicana de Estudios del Cuaternario (UMEC) y Universidad Nacional Autónoma de México (UNAM), Fondo de Cultura Económica, Ciudad de México.

VIDAL, A., 2002. Análisis de la cerámica utilitaria en un sitio agroalfarero temprano en la Puna de Catamarca. Tesis de licenciatura, Universidad de Buenos Aires, Buenos Aires.

VIGLIANI, S., 1999. Cerámica y asentamiento. Sistema de producción agrícola Belén-Inca. Tesis de licenciatura, Universidad de Buenos Aires, Buenos Aires.

ZAGORODNY, N., B. BALESTA y M. MOROSI, 2008. Caracterización composicional de la cerámica funeraria de La Ciénaga. En Problemáticas de la Arqueología Contemporánea, A. Austral y M. Tamagnini (Comps.), tomo II: 253-26o. Universidad Nacional de Río Cuarto, Río Cuarto. 